\title{
IUCN
}

\section{IUCN Green Status of Species}

A global standard for measuring species recovery and assessing conservation impact

Prepared by the IUCN SSC Species Conservation Success Task Force Version 2.0

\section{FSC \\ C. \\ re: wild

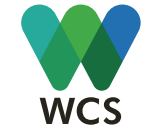 \\ UNIERSTY
OXFORD




\section{About IUCN}

IUCN is a membership Union uniquely composed of both government and civil society organisations. It provides public, private and non-governmental organisations with the knowledge and tools that enable human progress, economic development and nature conservation to take place together.

Created in 1948, IUCN is now the world's largest and most diverse environmental network, harnessing the knowledge, resources and reach of more than 1,400 Member organisations and some 18,000 experts. It is a leading provider of conservation data, assessments and analysis. Its broad membership enables IUCN to fill the role of incubator and trusted repository of best practices, tools and international standards.

IUCN provides a neutral space in which diverse stakeholders including governments, NGOs, scientists, businesses, local communities, indigenous peoples organisations and others can work together to forge and implement solutions to environmental challenges and achieve sustainable development.

Working with many partners and supporters, IUCN implements a large and diverse portfolio of conservation projects worldwide. Combining the latest science with the traditional knowledge of local communities, these projects work to reverse habitat loss, restore ecosystems and improve people's well-being.

www.iucn.org

https://twitter.com/IUCN/ 


\section{IUCN Green Status of Species}

A global standard for measuring species recovery and assessing conservation impact

Prepared by the IUCN SSC Species Conservation Success Task Force Version 2.0 
The designation of geographical entities in this book, and the presentation of the material, do not imply the expression of any opinion whatsoever on the part of IUCN or other participating organisations concerning the legal status of any country, territory, or area, or of its authorities, or concerning the delimitation of its frontiers or boundaries.

The views expressed in this publication do not necessarily reflect those of IUCN or other participating organisations.

Prepared by the IUCN SSC Species Conservation Success Task Force. As approved at the 102nd meeting of the IUCN Council, Gland, Switzerland (01/12/2020).

IUCN is pleased to acknowledge the support of its Framework Partners who provide core funding: Ministry of Foreign Affairs of Denmark; Ministry for Foreign Affairs of Finland; Government of France and the French Development Agency (AFD); the Ministry of Environment, Republic of Korea; the Norwegian Agency for Development Cooperation (Norad); the Swedish International Development Cooperation Agency (Sida); the Swiss Agency for Development and Cooperation (SDC) and the United States Department of State.

This publication has been made possible in part by funding from UKRl's Natural Environment Research Council (NERC), WWF US, Re:wild (formally GWC), IUCN Species Survival Commission, the University of Oxford, Stony Brook University, and the Prince Albert Foundation (via the Cambridge Conservation Initiative).

\section{Published by: $\quad$ IUCN, Gland, Switzerland}

Produced by: IUCN SSC Species Conservation Success Task Force

Copyright: $\quad$ @ 2021 IUCN, International Union for Conservation of Nature and Natural Resources Reproduction of this publication for educational or other non-commercial purposes is authorised without prior written permission from the copyright holder provided the source is fully acknowledged.

Reproduction of this publication for resale or other commercial purposes is prohibited without prior written permission of the copyright holder.

Recommended IUCN (2021). IUCN Green Status of Species: A global standard for measuring species recovery and assessing conservation citation: impact. Version 2.0. Gland, Switzerland: IUCN.

ISBN: 978-2-8317-2094-4 (PDF)

DOI: https://doi.org/10.2305/IUCN.CH.2021.02.en

Cover photos: [front cover] Amazon Gladiator Frog, Hypsiboas boans, in Cocobolo Nature Reserve, Panama @ Robin Moore [back cover] Magnolia orbiculata at National Botanic Garden in Cuba @ Ernesto Teste

Layout by: Jessica Avanidhar, jessavanidhar.com 


\section{Table of Contents}

Acknowledgments .......................................................................................... iv

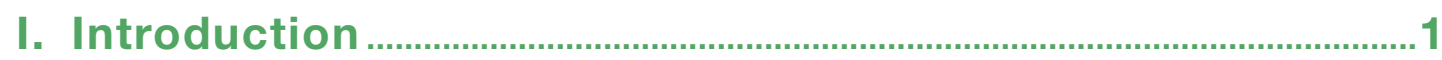

II. Preamble

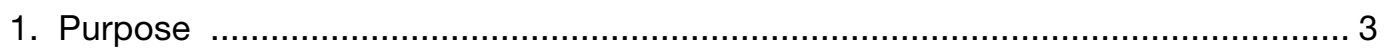

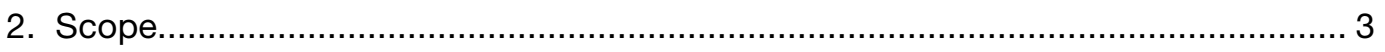

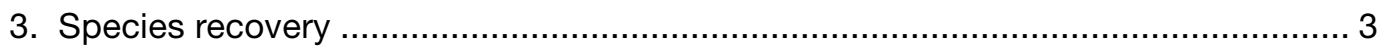

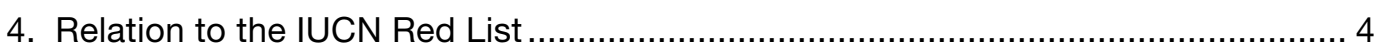

5. Relation to conservation planning and priorities ................................................ 4

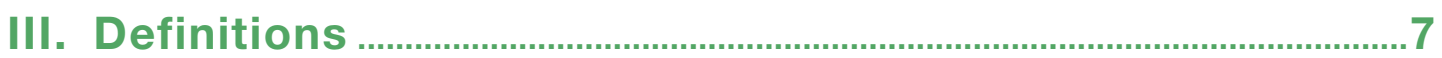

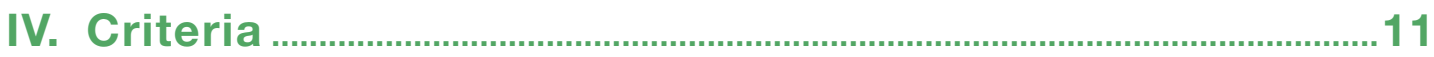

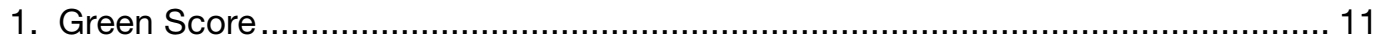

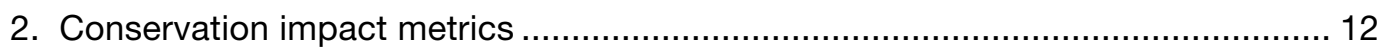

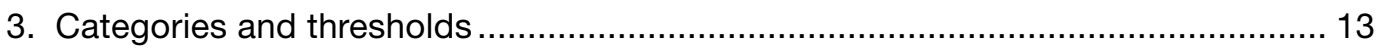

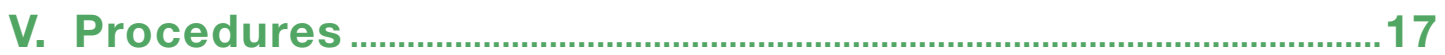

1. Determining range

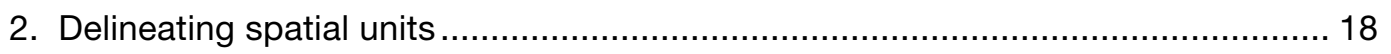

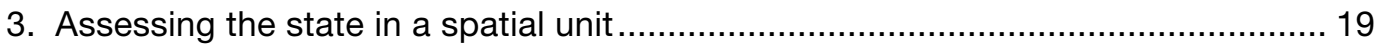

4. Developing the Counterfactual Current scenario ................................................. 20

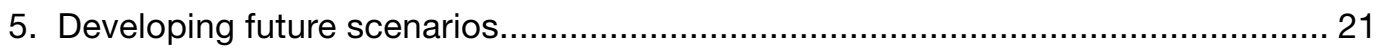

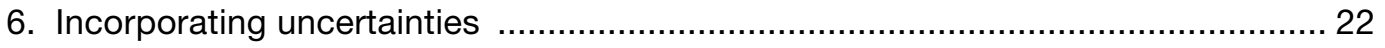

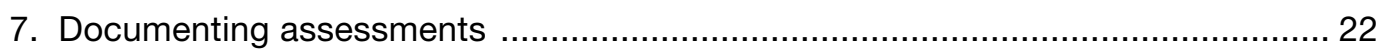

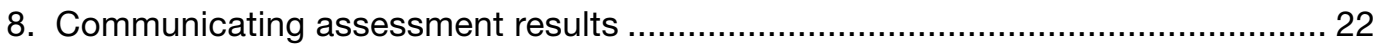

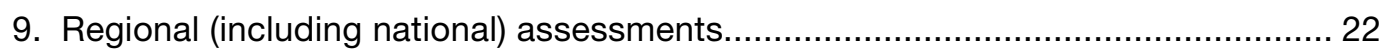

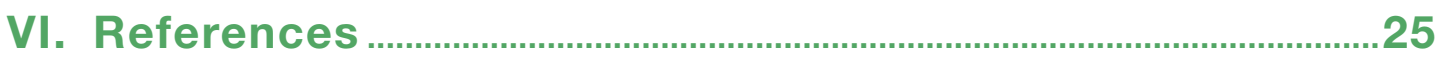




\section{Acknowledgments}

IUCN gratefully acknowledges the dedication and efforts of the Species Conservation Success Task Force (SCSTF) in proposing the methods of the IUCN Green Status of Species and conducting rigorous testing of the proposed methods via consultations, workshops, and scientific studies. The SCSTF was co-chaired by Barney Long and Elizabeth Bennett, and was coordinated by Molly Grace. The members of the SCSTF core group were: Resit Akçakaya, Craig Hilton-Taylor, Mike Hoffmann, E.J. Milner-Gulland, Richard Jenkins, Ana Nieto, and Richard Young; wider SCSTF members were: Thomas Brooks, Anna Heath, Simon Hedges, David Keith, David Mallon, Erik Meijaard, Ana Rodrigues, Jon Paul Rodríguez, PJ Stephenson, and Simon Stuart. Special thanks are due to Resit Akçakaya, who took a leading role in the scientific development of the method.

To develop the method for the Green Status of Species, the SCSTF formed several working groups, which included both SCSTF members and external experts: a Targets and Benchmarks Working Group, led by PJ Stephenson; a Scenarios Working Group, led by E.J. Milner-Gulland; a Viability and Functionality Working Group, led by Resit Akçakaya; a Spatial Units Working Group, led by Elizabeth Bennett and Molly Grace; a Technical Issues Working Group, led by Craig Hilton-Taylor and Michael Hoffmann; and a Communication Working Group, led by Barney Long. Non-SCSTF members who participated in these groups were: Alison Boyer, Joe Bull, Onnie Byers, Cheli Cresswell Sinclair, Axel Hochkirch, Ackbar Joolia, Phil McGowan, Elaine Paterson, Eric Sanderson, Carrie Stengel, Ricardo Tejada, Jessica Welch, and the Red List Technical Working Group.

The SCSTF carried out extensive piloting of the Green Status of Species method with 181 species, overseen by Molly Grace. Over 200 SSC Specialist Group members and independent species experts volunteered their time and knowledge to conduct pilot species assessments, which were critical for refining the method. Fifteen of these assessments were sponsored by National Geographic and led by PJ Stephenson. A consultation of potential end-users was led by Nigel Dudley and Hannah Timmins of Equilibrium Research; the feedback from representatives of business, policy, NGOs, and more greatly helped streamline the method (Dudley \& Timmins, 2021). A terminology consultation with IUCN regional offices, IUCN SSC regional representatives, zoos and aquaria, and journalists was conducted by Sarah Leonard, to facilitate effective communication of the final product.

The work of the SCSTF was made possible through generous financial support from the UKRI's Natural Environment Research Council (NERC), WWF US, Re:wild (formally GWC), IUCN Species Survival Commission, the University of Oxford, Stony Brook University, and the Prince Albert Foundation (via the Cambridge Conservation Initiative). We thank the following organisations for financially supporting SCSTF workshops: NERC, Fondation Franklinia, the Prince Albert Foundation (via the Cambridge Conservation Initiative), and the Oxford Knowledge Exchange Seed Fund. Several organisations contributed by allowing SCSTF members to contribute on salaried time: Wildlife Conservation Society, the Zoological Society of London, and Durrell.

Finally, IUCN is indebted to the Commission members and representatives of Member Organisations who participated in consultation workshops or who submitted comments and suggestions during the review process for this Standard. This combined input has resulted in a far more robust, user friendly and widely applicable system. 


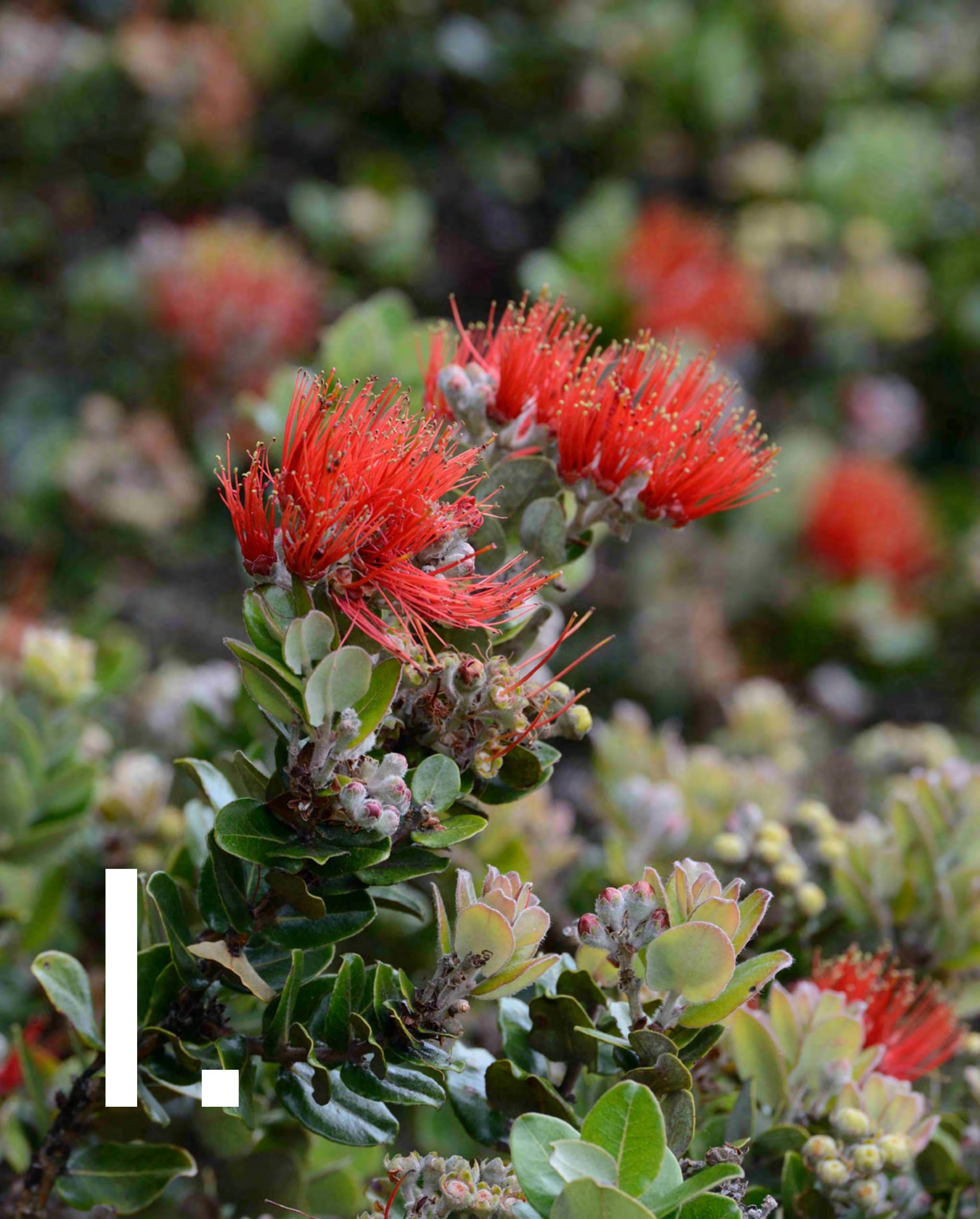




\section{Introduction}

The decline of many species towards extinction has largely focused conservation efforts on ensuring that species remain extant. However, conservationists have long recognised the need to complement this by aiming to recover depleted populations throughout a species' range and to restore species to ecosystems from which they have been extirpated.

Recognising this need, the International Union for Conservation of Nature (IUCN) called for the development of objective criteria for Green Lists of Species, Ecosystems and Protected Areas (IUCN \& WCPA, 2017). Resolution WCC-2012RES-41 of the 2012 World Conservation Congress requested that "the Species Survival Commission (SSC)... conduct international scientific consultations to develop objective, transparent and repeatable criteria for Green Lists that systematically assess successful conservation of species."

In response, the Species Survival Commission convened a task force on assessing conservation success under the auspices of the IUCN Red List Committee to oversee the development of this Standard. The Task Force developed a framework for measuring species recovery and conservation impact (Akçakaya et al., 2018) ${ }^{1}$, which proposed a definition of a fully recovered species based on viability, functionality, and representation, and defined four metrics to quantify the importance of conservation for a species. This framework was tested across a range of taxa between 2018 and 2021 to ensure wide applicability, and resulting changes are captured in this Standard.

To ensure full understanding of IUCN Green Status of Species assessments, it is very important to refer to the latest versions of all the following documents:

1. Background and Guidelines for the IUCN Green Status of Species, which accompanies this Standard, and which will be periodically updated (referred to in the remainder of this document as Background and Guidelines);

2. IUCN Red List Categories and Criteria (IUCN, 2012a);

3. Guidelines for Using the IUCN Red List Categories and Criteria (IUCN, 2019);

4. Guidelines for Application of IUCN Red List Criteria at Regional and National Levels (IUCN 2012b).

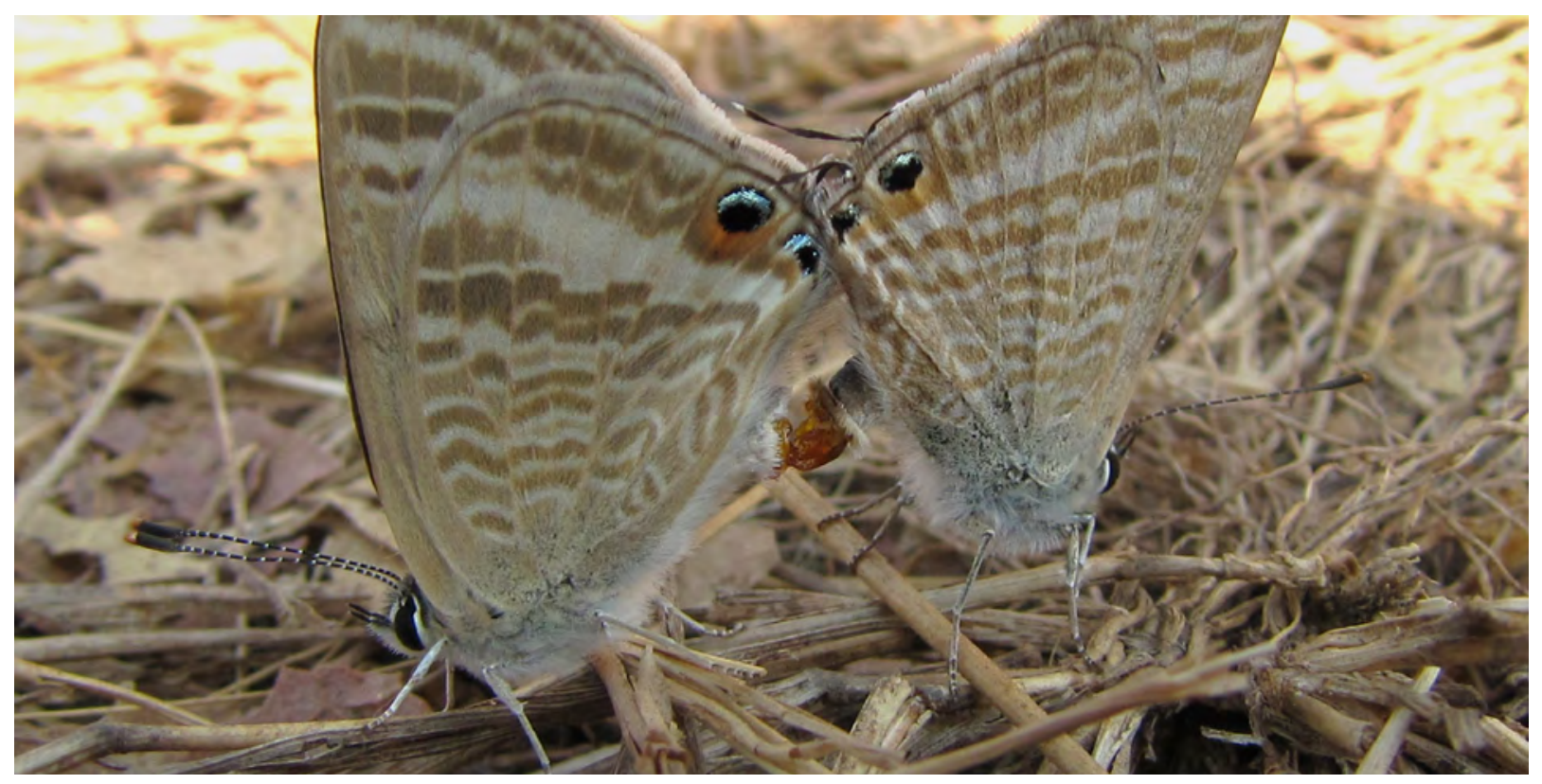

Mating of Pea Blue (Lampides boeticus) @) Neha Mujumdar

1 Akçakaya et al. (2018) is considered to be version 1.0 of the Standard; the document you are reading is version 2.0 and reflects the changes made in response to the testing and consultations that took place between 2018 and 2020. 


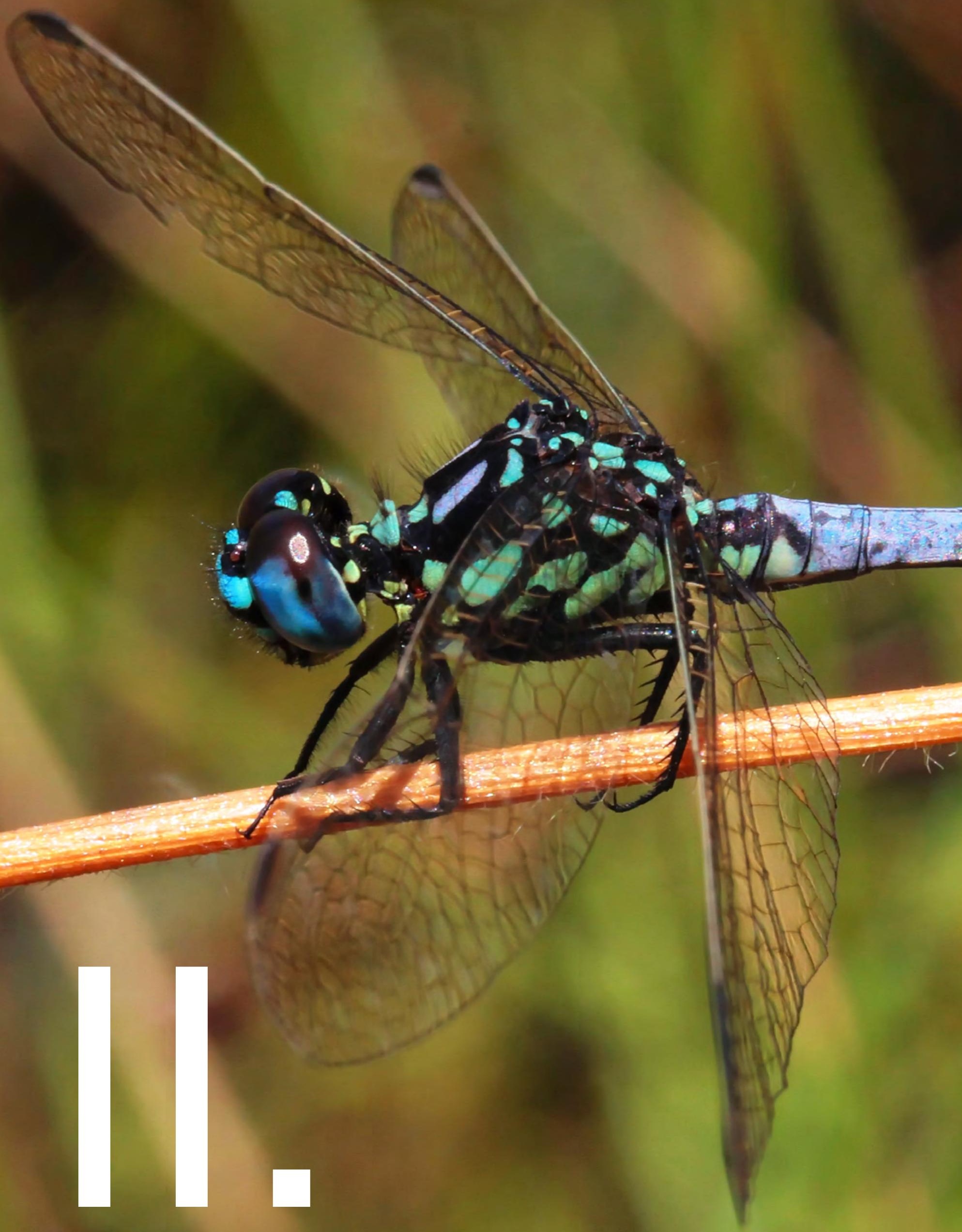




\section{Preamble}

\section{Purpose}

The IUCN Green Status of Species has five main objectives:

i. To provide a standardised framework for measuring species recovery;

ii. To recognise conservation achievements;

iii. To highlight species whose current conservation status is dependent on continued conservation actions;

iv. To forecast the expected conservation impact of planned conservation action; and

v. To elevate levels of ambition for long-term species recovery.

These objectives together encourage conservation towards species recovery, throughout a species' range. They are represented by a Species Recovery Score, and by four conservation impact metrics (Conservation Legacy, Conservation Dependence, Conservation Gain, Recovery Potential), which are quantified as differences between the Green Score of the species in different time steps or under different scenarios.

\section{Scope}

The definitions and metrics of the Green Status of Species can be applied to any species except microorganisms. Species can be assessed under this protocol regardless of their Red List category, and regardless of whether they have been subject to conservation measures. For practical reasons (see section V.3), Green Status of a species should be assessed after, or concurrently with, the Red List assessment of that species.

\section{Species recovery}

In this Standard, a Fully Recovered species is defined based on viability, functionality, and representation (see definition in section III). Viability is the first requirement that is essential but not sufficient for recognising a species as recovered. To be considered Fully Recovered, a species must also exhibit its ecological interactions, functions, and other roles in the ecosystem, and occur in a representative set of ecosystems and communities throughout its range. The viability and functionality aspects are addressed in the assessment of the state of the species' population in each spatial unit (see sections IV.1, V.3.c and V.3.d), and the representation aspect is addressed by making the assessment in all spatial units across the species' range (see sections IV.1 and V.2). The definition based on these characteristics is used to measure a species' recovery, expressed as the Green Score, which in turn is used to define four conservation impact metrics to quantify the importance of conservation for the species (see section III, Definitions).

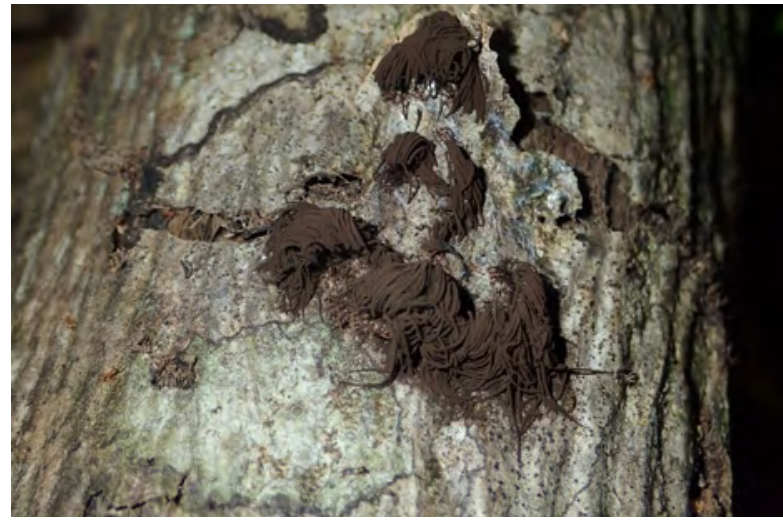

Stemonaria longa in Guadeloupe (c) Alain Michaud

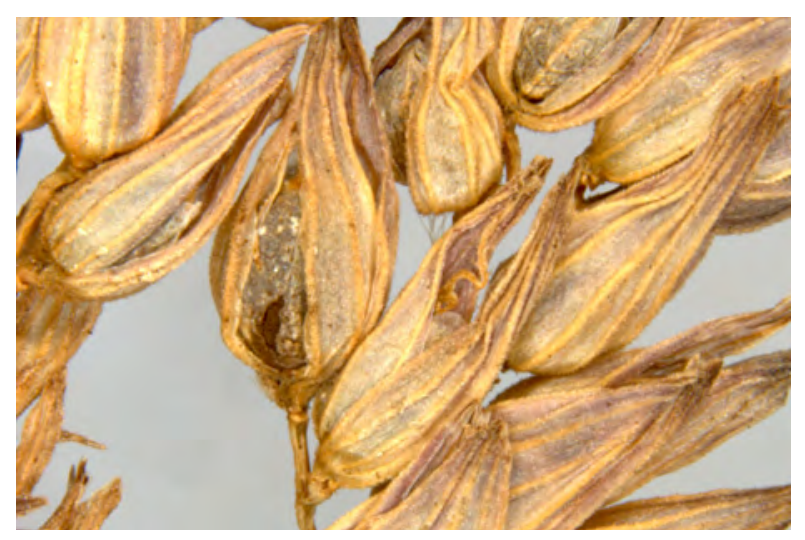

Ustilago suddiana @ C. M. Denchev 


\section{Relation to the IUCN Red List}

Green Status assessments are not an alternative to extinction risk assessments through the IUCN Red List, but provide complementary information. The results of a Green Status assessment (Species Recovery Score and Category, and the conservation impact metrics and categories) should be considered together with the IUCN Red List category of species. There is not a simple and general relationship between the Red List status and Green Status of species. Species that have been recovering may still be threatened; species that have not recovered may not be threatened; and species with high values of the conservation impact metrics may or may not be at risk of extinction. The Red List and Green Status provide separate but related and complementary assessments of the conservation status of a species.

Some issues that are relevant for Green Status assessments, for example terms used to define viability, are covered in the Red List Guidelines; thus, assessors should also consult the most recent version of the Guidelines for Using the IUCN Red List Categories and Criteria (IUCN Standards and Petitions Subcommittee, 2019), as they are updated on a regular basis.

\section{Relation to conservation planning and priorities}

Green Status Assessments are not meant to replace the process for establishing recovery objectives, targets and goals, which is a part of the conservation action planning process that brings together all stakeholders in the planning process, and that is the ideal and appropriate venue to set conservation targets and priorities. Instead, Green Status Assessments aim to be a useful and integral part of this planning process, and to reflect the targets and aspirations which are set within this process. Species conservation or recovery plans are often developed with conservation measures and actions covering a relatively short time horizon (e.g. five or ten years); this is reflected in the Conservation Gain metric. These plans are usually nested within a longer-term vision, which may often be 50-100 years. The Recovery Potential metric is aligned with this long-term vision. The time frame for Recovery Potential is set at 100 years, to link explicitly to the vision statements in many conservation strategies and action plans. The Recovery Potential should ideally be based on the long-term vision statement of a recognised action planning process, involving considered and appropriate stakeholder engagement. Conservation planning processes also often build upon past conservation actions and evaluations of conservation impacts in different parts of the species' range, as well as the species' short-term conservation needs. These are captured in the Conservation Legacy and Conservation Dependence metrics.

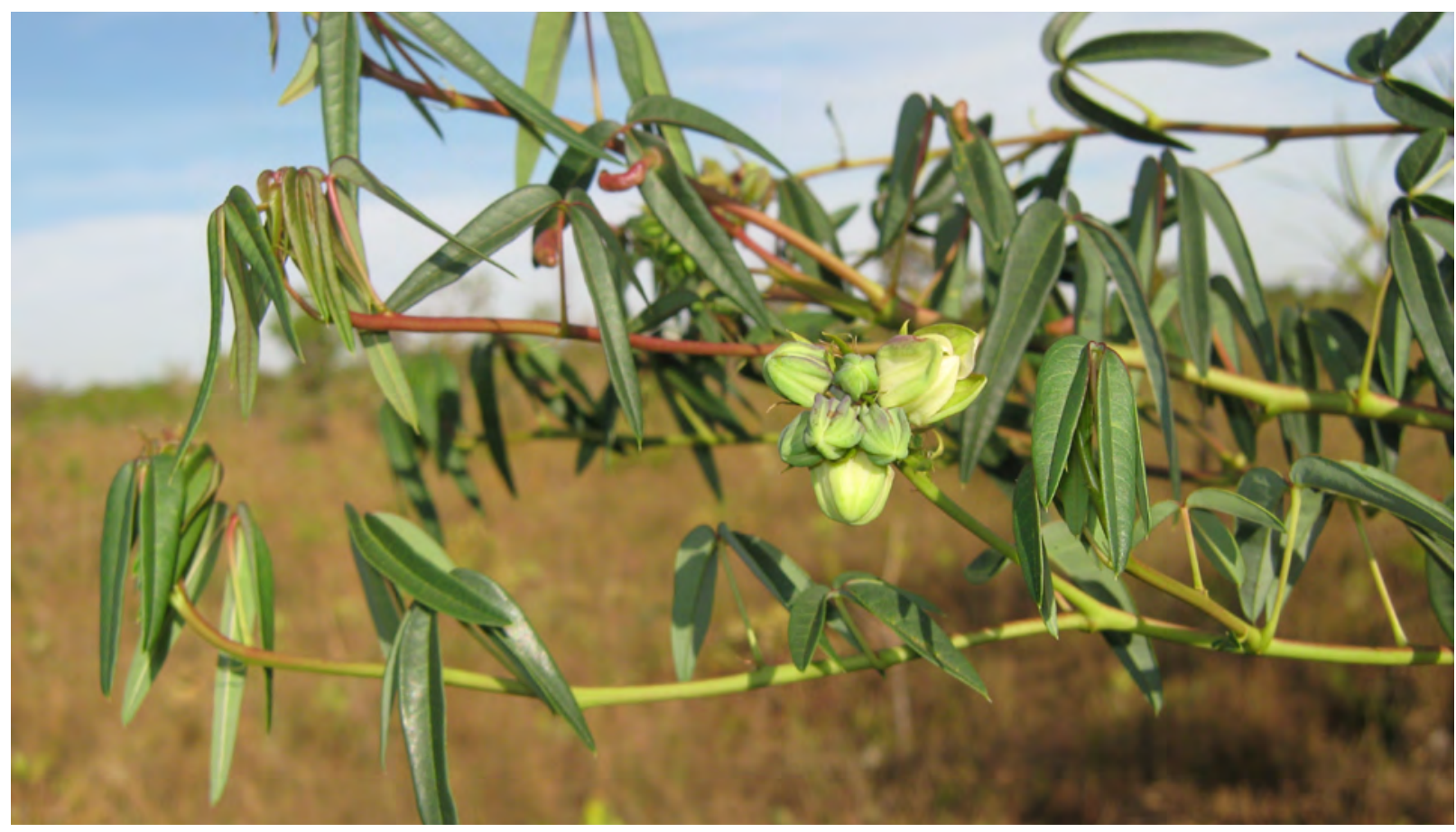

Manihot gracilis Pohl, a secondary genetic wild relative of cassava (M. esculenta subsp. esculenta), in Brazil @ Marcelo Simon 


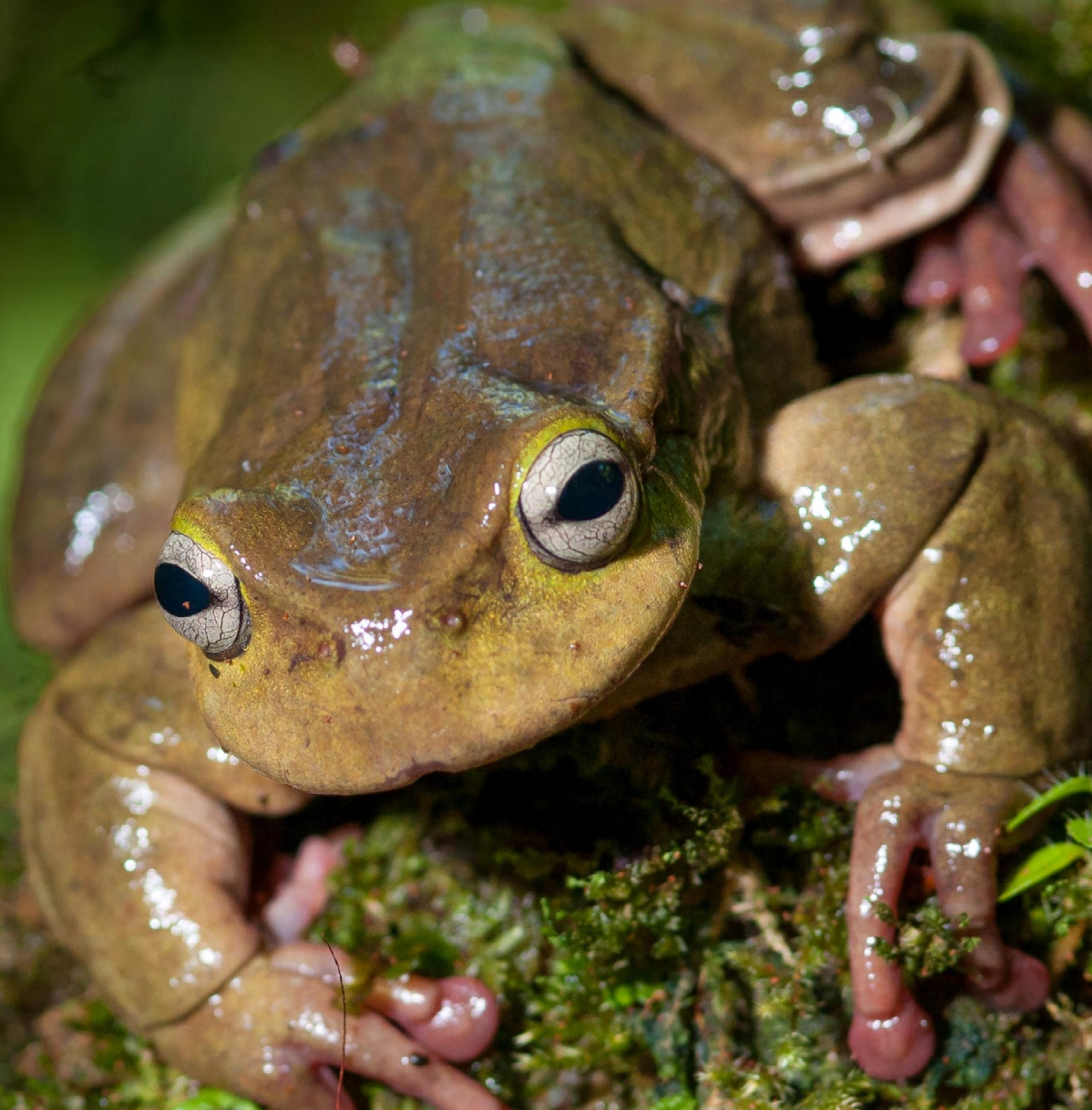

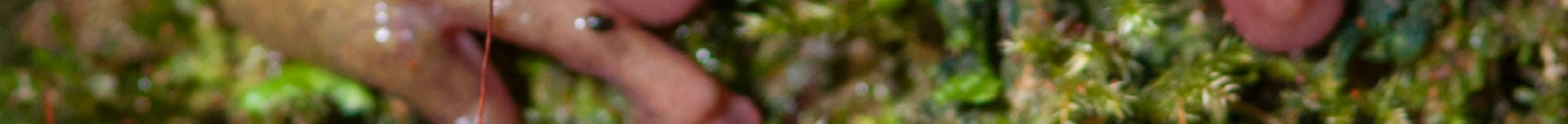

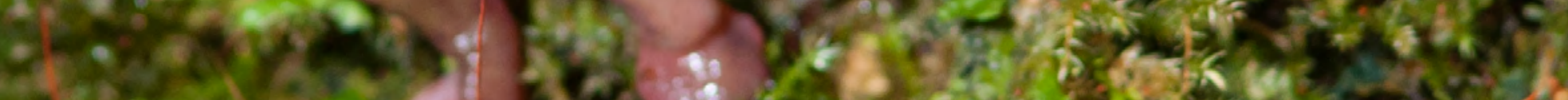
(1.

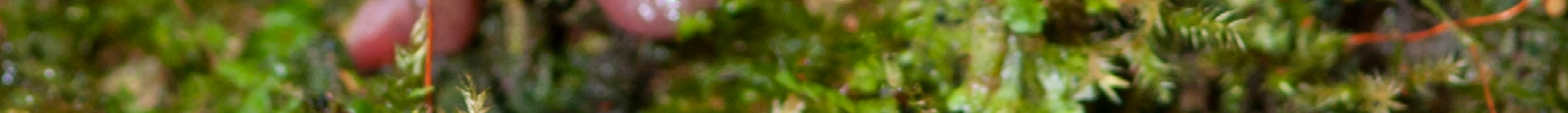

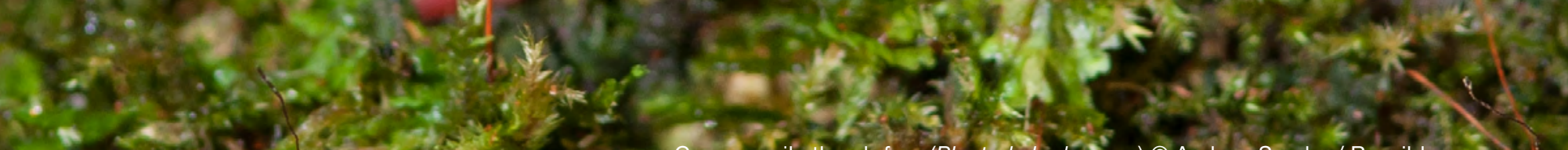

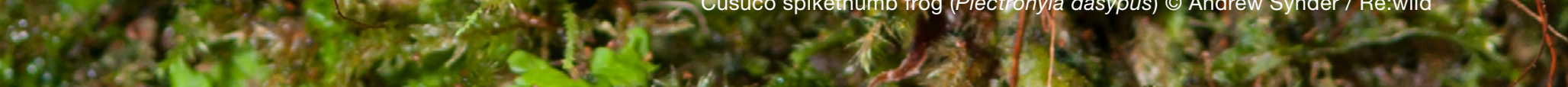

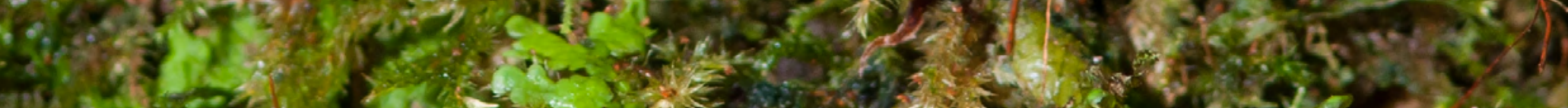




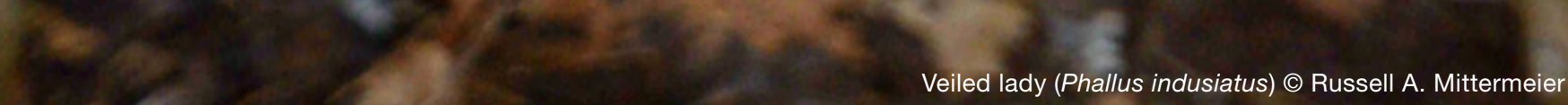




\begin{tabular}{ll}
\hline Absent (state of a spatial unit) & A species is said to be Absent in a given spatial unit if it does not occur there \\
in the wild, despite the spatial unit being a part of the species' range. This is \\
one of four possible states for a spatial unit population (with Present, Viable, \\
and Functional).
\end{tabular}

Conservation action

Any human activity for which biodiversity conservation is a stated intention, even if the activity is not for the sole, or primary, purpose of biodiversity conservation, and even if its budget is from sources other than conservation programmes.

Conservation Dependence

A conservation impact metric that measures the impact of ongoing conservation actions, defined as the predicted change in the Green Score of the species in the short-term future (10 years) if all conservation actions were to cease, beginning today. The metric is calculated as the difference between the Current (or Current Baseline) Green Score and the Future-without-conservation Green Score (see section IV.1, IV.2, V.5.c, and Figure 1).

Conservation Gain

A conservation impact metric that measures the impact of ongoing and planned conservation actions, defined as the predicted change in the Green Score of the species in the short-term future (10 years) if ongoing and planned future actions are implemented effectively. The metric is calculated as the difference between the Current (or Current Baseline) Green Score and the Future-with-conservation Green Score (see section IV.1, IV.2, V.5.c, and Figure 1).

Conservation Legacy

A conservation impact metric that measures the impact of conservation actions that have been conducted to date, defined as the difference between the species' Current Green Score and its Counterfactual Current Green Score (see section IV.1 and Figure 1).

Conservation impact category

Each conservation impact metric can be expressed as either a percentage or using categories. For each of the four conservation impact metrics, the categories are: Negative, Zero, Low, Medium, High, or Indeterinate (see section IV.3).

Conservation impact metric

One of four measures of the importance of conservation actions for the species: Conservation Legacy; Conservation Dependence; Conservation Gain; and Recovery Potential. Each metric is calculated as the difference between two Green Scores.

Counterfactual

A hypothetical scenario of what the status of the species would have been today in the absence of past conservation actions; it is used to determine the Counterfactual Current state in each spatial unit, and the Counterfactual Current Green Score (see section IV.1)

Ecological function of a species

The totality of the species' interactions, determining its influence on, or contribution to, ecosystem processes, and the patterns of intra-specific interactions, behaviour and social dynamics that are characteristic of that species (see section V.3.d)

\section{Ecological functionality of a population}

Expected additional range
The extent to which the population in a spatial unit fulfils the ecological function or functions of the species in a particular time and place (e.g. a spatial unit), as determined by its size, density and demographic structure (see section V.3.d).

The areas that are strongly expected to become suitable and inhabited by the species in the next 100 years, taking into account range shifts resulting from climate change and other global and local processes, as well as conservation translocations (IUCN Standards and Petitions Committee, 2019). 
A species that is viable and ecologically functional in every part of its range. The Green Score is defined relative to this condition. This definition and the conservation impact metrics that are based on it apply not only to species that have previously declined, but also to taxa that have not declined (irrespective of whether or not they have been the focus of conservation so far). However, taxa that fit this definition but have not benefitted from conservation are categorised as Non-Depleted. A Fully Recovered (or Non-Depleted) species has a Green Score of $100 \%$.

Functional (state of a spatial unit) A spatial unit population is said to be Functional if it fulfils the ecological function(s) of the species; that is, it has the attributes (including, for example, abundance, density and demographic structure) that enable it to interact with other species, contribute to ecosystem processes, and/or display patterns of intra-specific interactions, behaviours and social dynamics that are characteristic of the species. This is one of four possible states for a spatial unit population (with Absent, Present, and Viable). See section V.3.d and Background and Guidelines section 4.5 .

\section{Green Score}

A numerical value between $0 \%$ and $100 \%$, representing how close the species is to being Fully Recovered. A value of $0 \%$ means the species is Extinct or Extinct in the Wild, and $100 \%$ means it is Fully Recovered. The Green Score is calculated based on the state (Absent, Present, Viable or Functional) in each spatial unit, and can be calculated for past, current, short-term future, and longterm future time periods, and for alternative scenarios (i.e. with conservation at various levels (past, current, planned, aspirational), or without conservation). These different Green Scores are used to calculate the conservation impact metrics for each species, and species are placed in categories based on these four metrics.

\section{Indigenous range}

The distribution of the species, generated from current and historical (written or verbal) records, or physical evidence of its occurrence, accounting for all known, inferred or projected sites of occurrence (IUCN, 2012a), including past conservation translocations (IUCN Standards and Petitions Committee, 2019) but not including vagrancies. Where direct evidence is inadequate to confirm previous occupancy, the existence of suitable habitat within ecologically appropriate proximity to observed range may be taken as adequate evidence of previous occupation (IUCN SSC, 2013).

Parts of the range See 'spatial unit.'

\section{Present (state of a spatial unit)}

A spatial unit population is said to be Present if the species occurs but is not Viable or Functional in the spatial unit. This is one of four possible states for a spatial unit population (along with Absent, Viable and Functional).

The spatial distribution of the species, comprising its indigenous range and expected additional range. For Conservation Legacy, the range is defined only as the indigenous range. For the other three conservation impact metrics, which involve the future of the species, the range can be defined as the combination of its indigenous range and expected additional range, depending on when the expected additional range is expected to become occupied. See section V.1.b. 
A conservation impact metric that quantifies conservation aspiration or ambition, defined as the maximum plausible improvement in the status of the species with sustained conservation efforts and conservation innovation over the longterm (100 years). See section IV.1 and Figure 1.

Rescue effect
The process by which the extinction risk in a spatial unit is decreased because of propagules immigrating from other spatial units. A propagule is a living entity capable of dispersal and of producing a new mature individual (e.g. a spore, seed, fruit, egg, larva, or part of or an entire individual). Gametes and pollen are not considered propagules in this context (IUCN, 2012b).

Spatial unit

A subdivision of the species' range in which the state of the species is assessed as Absent, Present, Viable, or Functional. See 'Delineating spatial units' in section $\mathrm{V}$, below.

Spatial unit population The set of individuals of a species in a spatial unit (cf. 'population' in IUCN (2012a) and IUCN (2019)). Also referred to as 'population in a spatial unit.'

\section{Species Recovery Category}

A measure of how close the species is to Fully Recovered, based on the Species Recovery Score and the thresholds stated in section IV.3.

Species Recovery Score

A measure of how close the species is to Fully Recovered, based on the observed, estimated, inferred, or suspected condition of the species at the time of assessment (shown in Figure 1 as the Current Green Score). It can be plotted as a function of time, based on condition at each time step, depicting the species' progress (or otherwise) towards full recovery.

State

The condition of the species in a spatial unit, assessed as one of four ordinal categories: Absent, Present, Viable, and Functional. These categories are assigned weights (see section V.3), which are combined, using Equation 1 below, to give the Green Score. State can be determined for past, current, short-term future, and long-term future time periods, and for alternative scenarios (with and without conservation). States are in a nested hierarchy, so that a spatial unit population that is assessed as Viable is also Present by definition, and a spatial unit population that is assessed as Functional is by definition also Viable and Present. Therefore a score of Functional can only be applied to spatial units that are both Viable and Functional (but see section V.3.d).

Subpopulation

Subpopulations are defined as geographically or otherwise distinct groups in the population between which there is little demographic or genetic exchange (typically one successful migrant individual or gamete per year or less) (IUCN, 2012a; see IUCN Standards and Petitions Committee (2019) for guidance).

Viable (state of a spatial unit)
A spatial unit population is said to be Viable if the species has a low risk of extirpation in the spatial unit, as determined by the regional IUCN Red List category. See Section V.3 for details. 


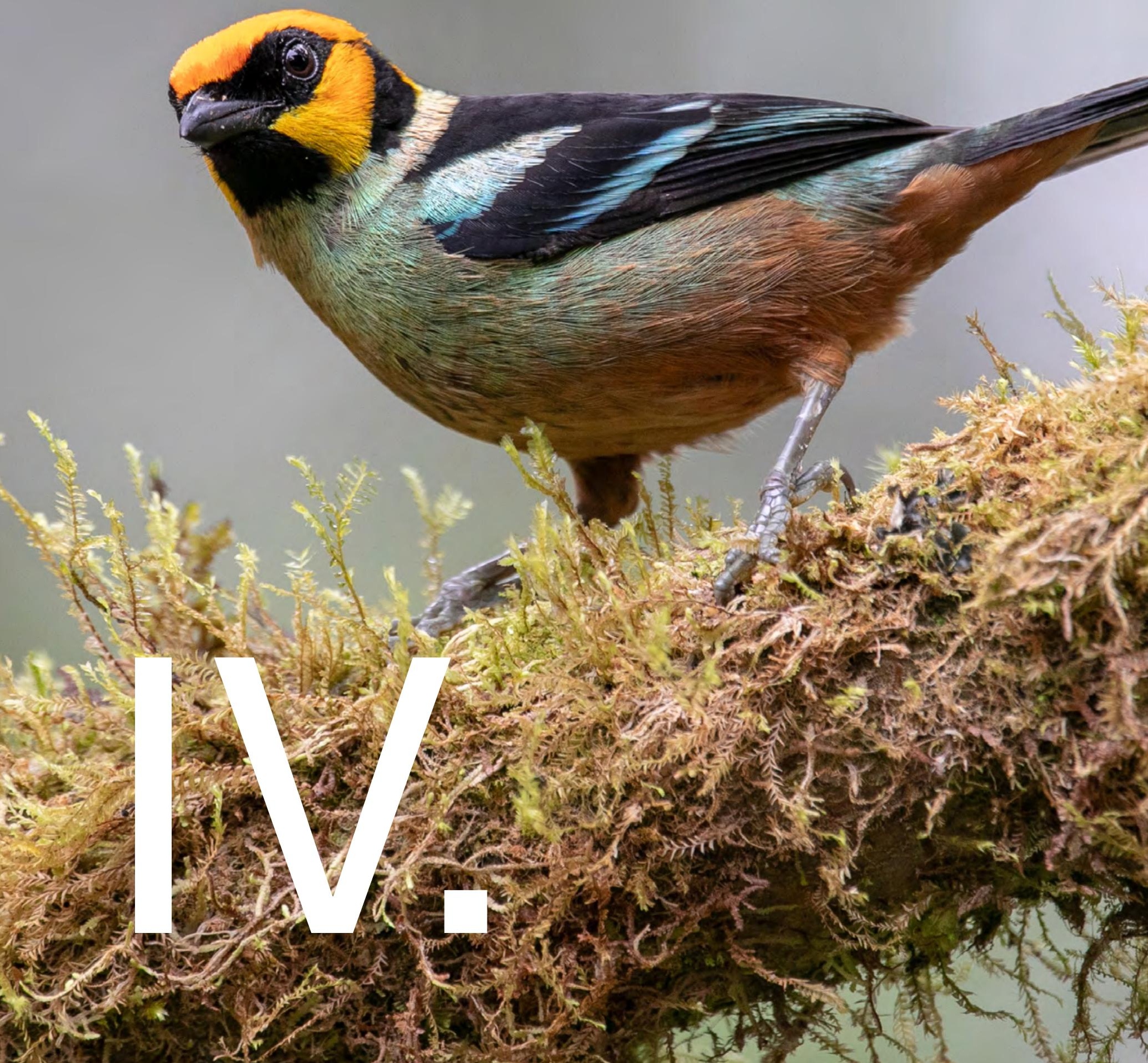




\section{Criteria}

\section{Green Score}

The state in each spatial unit is assessed as one of four ordinal categories: Absent, Present, Viable, and Functional (see section V.3. for details). A Green Score (G) for the species is obtained, based on the states in all spatial units, with the equation (Equation 1):

$$
G=\frac{\sum_{s} W_{s}}{W_{F} \times N} \times 100
$$

where $s$ is each spatial unit, $W_{S}$ the weight of the state (Absent, Present, Viable, or Functional) in the spatial unit, $W_{F}$ is the weight of the Functional state, and $\mathrm{N}$ is the number of spatial units. The denominator is the maximum possible score attained when all spatial units are assessed as Functional. Thus, a Green Score is calculated as a percentage of Fully Recovered. For Current and Counterfactual Current scores, the denominator is based on the number of spatial units in the indigenous range only (not including the expected additional range).

Different Green Scores are calculated based on current, short-term future, and long-term future time periods, and alternative scenarios, as listed in Table 1. Conservation impact metrics (e.g. Conservation Legacy, etc.) are calculated as differences between two Green Scores, as detailed in section V.

Table 1. Names and descriptions of the scenarios under which Green Score can be calculated. Note that all Green Status assessments require at least the 'Current' scenario; assessing conservation impact requires at least one other scenario.

\begin{tabular}{|ll|}
\hline Green Score & Scenario and time period \\
\hline Current & $\begin{array}{l}\text { The Green Score at the time of the assessment (the same as the Species Recovery Score at } \\
\text { that time). }\end{array}$ \\
\hline Counterfactual Current & $\begin{array}{l}\text { What the value of the Green Score would have been today in the absence of past conservation } \\
\text { actions. See section V.4. }\end{array}$ \\
\hline Current Baseline & $\begin{array}{l}\text { Predicted value of the Green Score in the short-term future (10 years), considering the likely } \\
\text { benefits of conservation actions that are currently in place or very likely to be in place within } 1 \\
\text { year. If the Current Baseline scenario is not specified, it is assumed to be the same as Current. } \\
\text { See section V.5.c. }\end{array}$ \\
\hline Future-with- conservation & $\begin{array}{l}\text { Predicted value of the Green Score in the short-term future (10 years), considering the likely ben- } \\
\text { efits of conservation actions that are currently in place or are planned for implementation during } \\
\text { this time window. See section V.5.a. }\end{array}$ \\
\hline Future-without-conservation & $\begin{array}{l}\text { Predicted value of the Green Score in the short-term future (10 years), assuming any ongoing } \\
\text { conservation actions stop today, and no new actions are implemented. See section V.5.b. }\end{array}$ \\
\hline Long-term Potential & $\begin{array}{l}\text { Predicted value of the Green Score in the long-term future (100 years), given sustained and effec- } \\
\text { tive conservation action and innovation. See section V.5.d. }\end{array}$
\end{tabular}




\section{Conservation impact metrics}

For any given species, four conservation impact metrics are calculated as a difference between two Green Scores (see Figure 1):
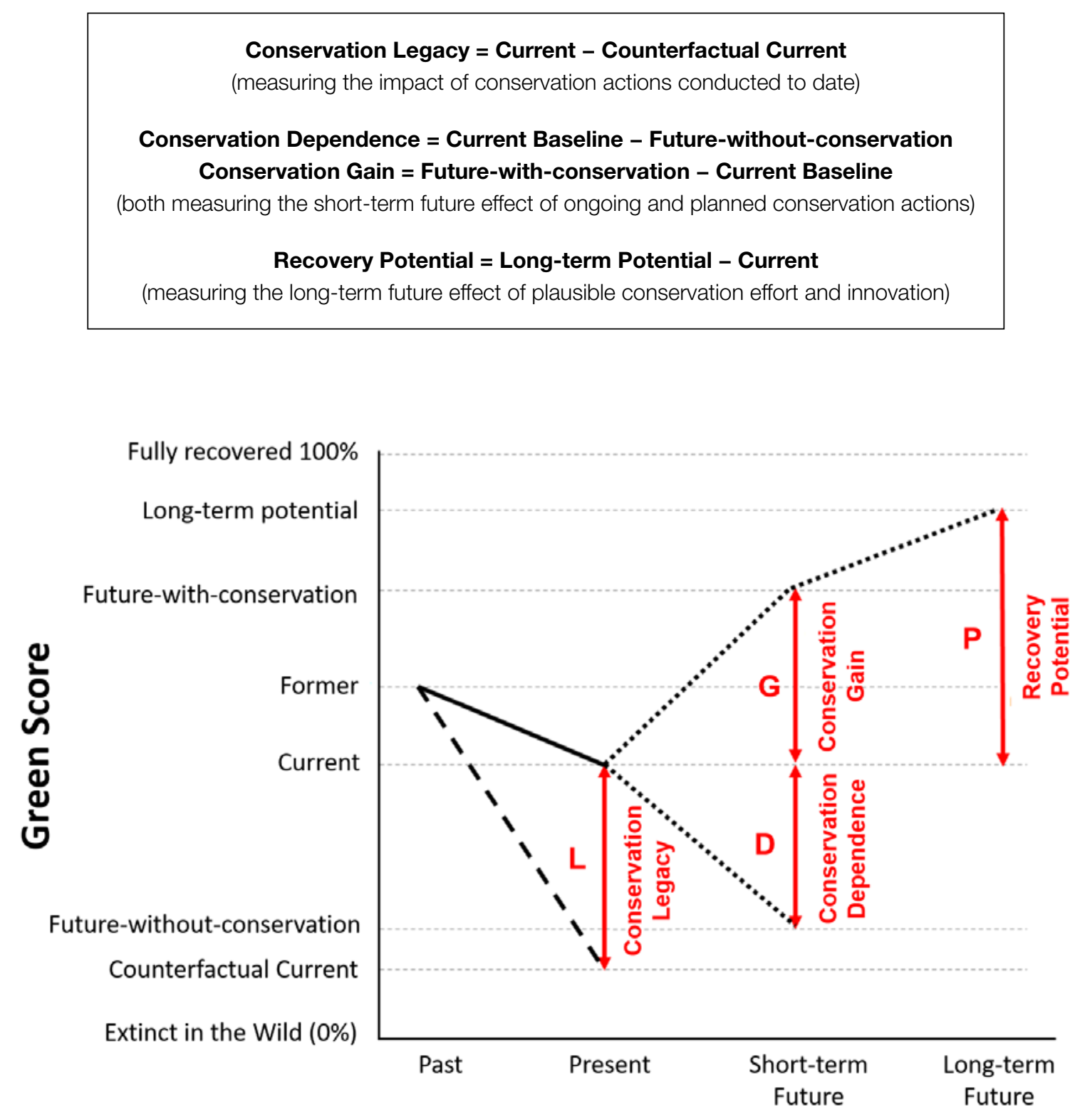

Time

Figure 1. Graphical representation of the conservation impact metrics as differences in the degree of recovery of the species (percent of Fully Recovered, measured as the Green Score; equation 1). Solid-black line: observed change in the Green score of the species. Dashed-black line: (counterfactual) past change expected in the absence of past conservation efforts. Dotted-black lines: future scenarios of change expected with and without current and future conservation efforts. Vertical arrows represent the conservation impact metrics: Conservation Legacy: Benefits of current and past conservation (current - counterfactual current); Conservation Dependence: Expected change in the short-term future in the absence of ongoing conservation (current baseline - future without conservation); Conservation Gain: Expected improvement in the short-term future with ongoing and planned conservation (future with conservation - current baseline); Recovery Potential: Possible improvement with long-term conservation (long-term potential - current). In this example, the Current Baseline (see section V.5.c) is assumed to have the same value as the Current score; see Background and Guidelines for the same graph with an alternative assumption. 


\section{Categories and thresholds}

For most purposes, the numerical (percentage) values of Species Recovery Score and the four conservation impact metrics, including their uncertainties (see section V.8, below), should be reported. However, categorical results may be more appropriate for some uses and audiences (see section V.8). Therefore, each metric is divided into categories, in order to (i) provide context and allow interpretation of the percentage value of the metric, and (ii) highlight cases of outstanding conservation impact, dependence, or potential.

The Species Recovery Score (SRS) can be reported either numerically (from 0\% for extinct to 100\% for Fully Recovered), or categorically. Species Recovery Categories are based on the best-estimate, minimum, and maximum values of the SRS $\left(\mathrm{SRS}_{\text {best }}\right.$, $\mathrm{SRS}_{\min }, \mathrm{SRS}_{\max }$, respectively) and the best-estimate value of Conservation Legacy $\left(\mathrm{L}_{\text {best }}\right)$, and are assigned according to the following rules, which are applied in the order listed until the condition given for a category is met (Table 2).

Table 2. Species Recovery Categories, based on the best-estimate, minimum, and maximum values of the Species Recovery Score $\left(\mathrm{SRS}_{\text {best }}, \mathrm{SRS}_{\min }, \mathrm{SRS}_{\max }\right.$, respectively):

\begin{tabular}{|ll|}
\hline Indeterminate & If $\left(\mathrm{SRS}_{\max }-\mathrm{SRS}_{\text {min }}\right)>40 \%$ \\
\hline Non-Depleted & If $\left(\mathrm{SRS}_{\text {best }}=100 \%\right)$ and $\left(\mathrm{L}_{\text {best }}=0 \%\right)$ \\
\hline Fully Recovered & If $\mathrm{SRS}_{\text {best }}=100 \%$ \\
\hline Slightly Depleted & If $\mathrm{SRS}_{\text {best }}>80 \%$ \\
\hline Moderately Depleted & If $\mathrm{SRS}_{\text {best }}>50 \%$ \\
\hline Largely Depleted & If $\mathrm{SRS}_{\text {best }}>20 \%$ \\
\hline Critically Depleted & If $\mathrm{SRS}_{\text {best }}>0 \%$ \\
\hline Extinct in the Wild & If $\mathrm{SRS}_{\text {best }}=0 \%$ \\
\hline
\end{tabular}


The category for each of the four conservation impact metrics is determined based on the conditions listed below (Tables 3-6). For each metric, the rules are applied in the order listed until the condition given for a category is met. In these conditions, best-estimate, minimum, and maximum values of the conservation impact metrics, and the best-estimate values of the Green Scores (such as Current and Counterfactual Current) are used. For each metric, the High category can be achieved in one of three ways: the numerical value is more than $40 \%$; the numerical value is small but represents avoidance of extinction; or the numerical value is small but is substantial compared with the best or medium estimate of the Current score. For information on the thresholds, see the Background and Guidelines.

Table 3. Conservation Legacy categories, based on the best-estimate, minimum, and maximum values of the Conservation Legacy metric $\left(L_{\text {best }}, L_{\min }\right.$, $L_{\max }$, respectively):

\begin{tabular}{|ll|}
\hline Indeterminate & If $\mathrm{L}_{\min }<0 \%$ and $\mathrm{L}_{\max }>40 \%$ \\
\hline High & $\begin{array}{l}\text { If } \mathrm{L}_{\text {best }}>40 \% \text {, or } \\
\mathrm{L}_{\text {best }}>0 \% \text { and Counterfactual Current }=0 \text { (i.e. extinction prevented), or } \\
\mathrm{L}_{\text {best }}>1 / 2 \cdot \text { Current (i.e. substantial legacy relative to current score) }\end{array}$ \\
\hline Medium & If $\mathrm{L}_{\text {best }}>10 \%$ \\
\hline Low & If $\mathrm{L}_{\text {best }}>0 \%$ \\
\hline Zero & If $\mathrm{L}_{\text {best }}=0 \%$ \\
\hline Negative & If $\mathrm{L}_{\text {best }}<0 \%$ \\
\hline
\end{tabular}

Note: The character $\cdot$ is the mathematical operator for multiplication (same as $\times$ )

Table 4. Conservation Dependence categories, based on the best-estimate, minimum, and maximum values of the Conservation Dependence metric $\left(D_{\text {best }}, D_{\min }, D_{\max }\right.$, respectively):

Indeterminate If $\mathrm{D}_{\min }<0 \%$ and $\mathrm{D}_{\max }>40 \%$

\begin{tabular}{|ll|} 
High & $\begin{array}{l}\text { If } D_{\text {best }}>40 \%, \text { or } \\
D_{\text {best }}>0 \% \text { and Future-without-conservation=0 (i.e. would go extinct without conservation), or } \\
D_{\text {best }}>1 / 2 \cdot \text { Current (or Current Baseline, if used) (i.e. substantial dependence relative to reference } \\
\text { score) }\end{array}$ \\
\hline Medium & If $D_{\text {best }}>10 \%$ \\
\hline Low & If $D_{\text {best }}>0 \%$ \\
\hline Zero & If $D_{\text {best }}=0 \%$ \\
\hline Negative & If $D_{\text {best }}<0 \%$ \\
\hline
\end{tabular}


Table 5. Conservation Gain categories, based on the best-estimate, minimum, and maximum values of the Conservation Gain metric $\left(G_{\text {best }}, G_{\min }, G_{\max }\right.$, respectively):

\begin{tabular}{|ll|}
\hline Indeterminate & If $\mathrm{G}_{\min }<0 \%$ and $\mathrm{G}_{\max }>40 \%$ \\
\hline High & $\begin{array}{l}\text { If } \mathrm{G}_{\text {best }}>40 \% \text {, or } \\
\mathrm{G}_{\text {best }}>0 \% \text { and Current }=0 \text { (i.e. would remain EW without conservation), or } \\
\mathrm{G}_{\text {best }}>1 \cdot \text { Current (or Current Baseline, if used) (i.e. substantial recovery relative to } \\
\text { reference score) }\end{array}$ \\
\hline Medium & If $\mathrm{G}_{\text {best }}>10 \%$ \\
\hline Low & If $\mathrm{G}_{\text {best }}>0 \%$ \\
\hline Negative & If $\mathrm{G}_{\text {best }}=0 \%$ \\
\hline
\end{tabular}

Table 6. Recovery Potential categories, based on the best-estimate, minimum, and maximum values of the Recovery Potential metric $\left(P_{\text {best }}, P_{\min }, P_{\max }\right.$, respectively):

\begin{tabular}{|ll|}
\hline Indeterminate & If $\mathrm{P}_{\text {min }}<0 \%$ and $\mathrm{P}_{\max }>40 \%$ \\
\hline High & $\begin{array}{l}\text { If } \mathrm{P}_{\text {best }}>40 \% \text {, or } \\
\mathrm{P}_{\text {best }}>0 \% \text { and Current }=0 \text { (i.e. would remain EW without conservation), or } \\
\mathrm{P}_{\text {best }}>2 \cdot \text { Current (i.e. substantial recovery relative to current score) }\end{array}$ \\
\hline Medium & If $\mathrm{P}_{\text {best }}>10 \%$ \\
\hline Low & If $\mathrm{P}_{\text {best }}>0 \%$ \\
\hline Zero & If $\mathrm{P}_{\text {best }}=0 \%$ \\
\hline Negative & If $\mathrm{P}_{\text {best }}<0 \%$ \\
\hline
\end{tabular}




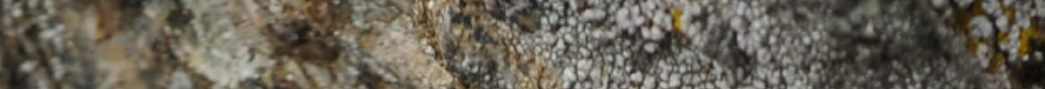

Q4a

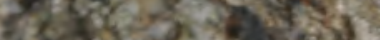

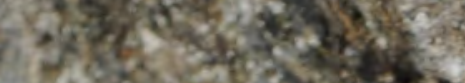

and $x$.

C.

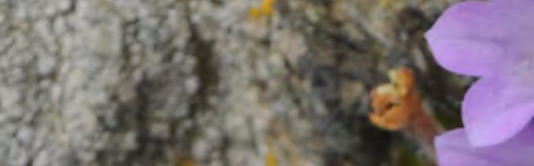

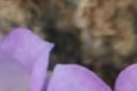

Bellflowers (Campanula mugeana) - O Hasanyildırm

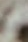

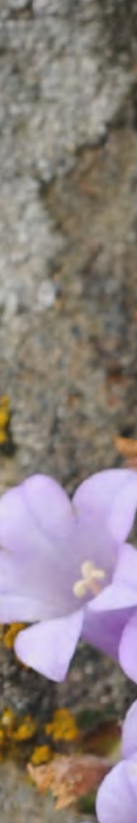
90

sonstis.

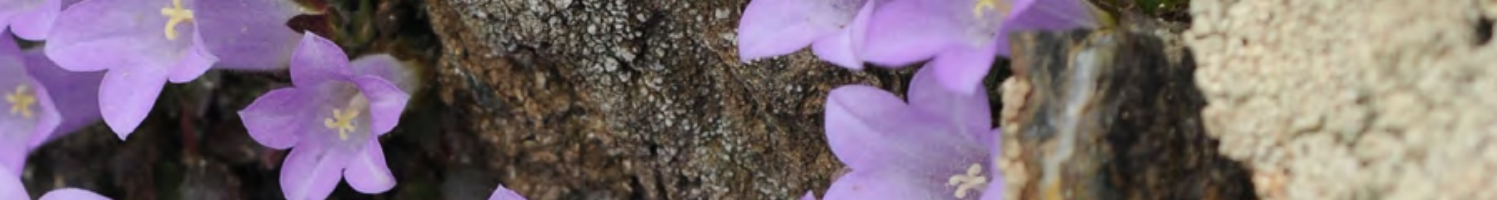

(3) 309

8.

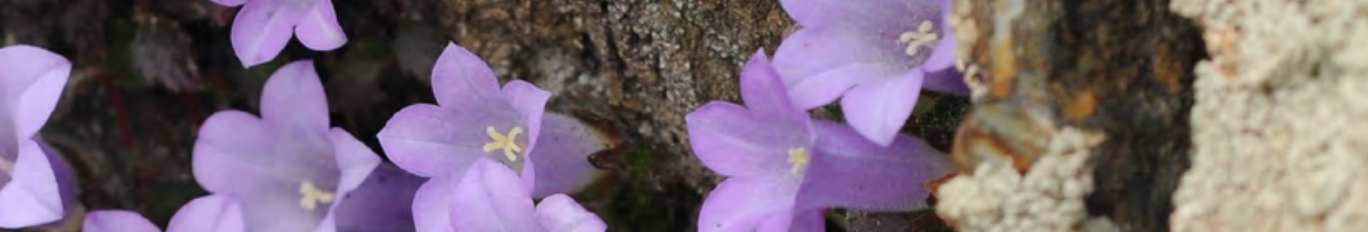

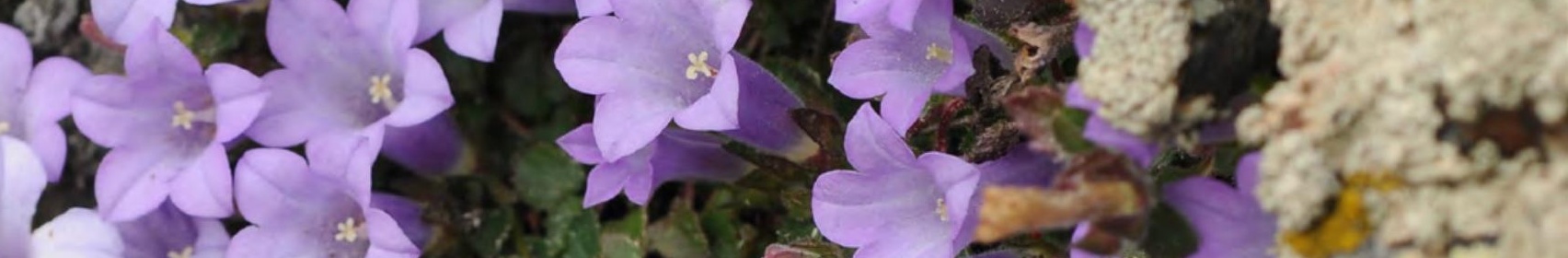

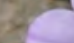

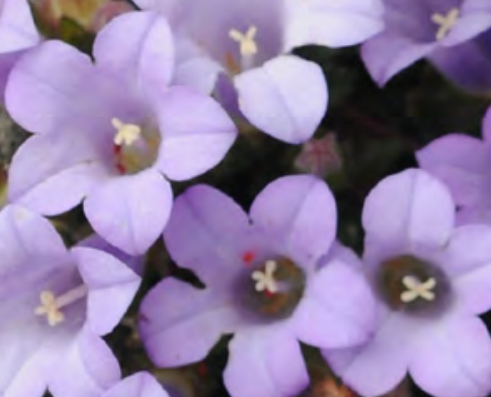

14

(4)

ext

8.

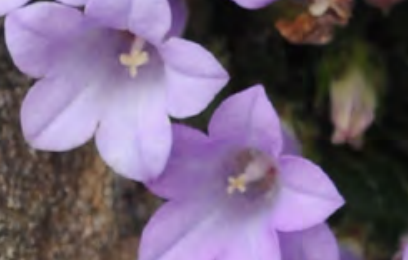

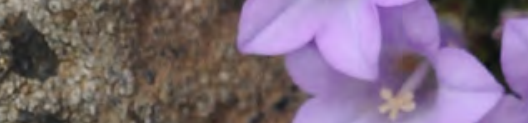

19.0

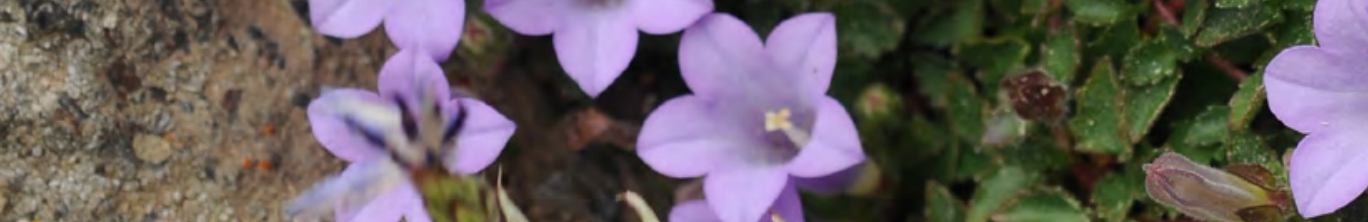

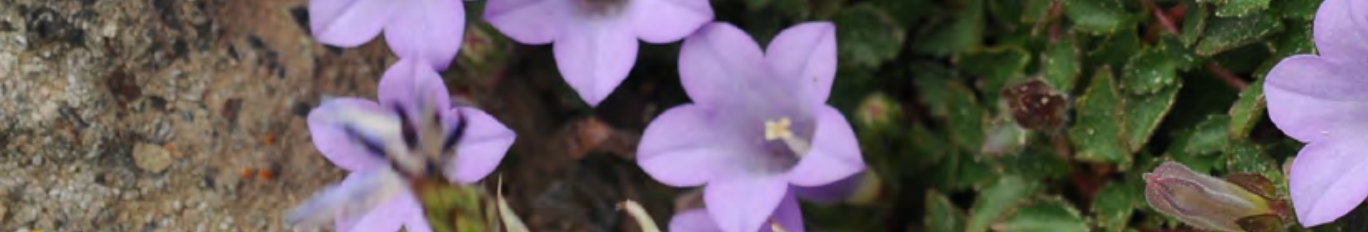

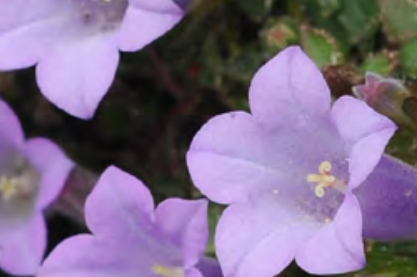

y

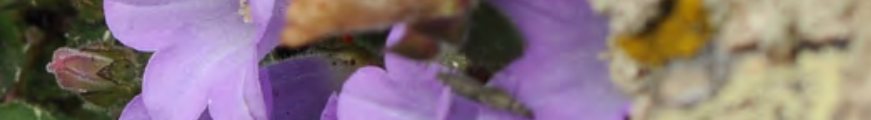

- fl $^{2} \leq \mathrm{con}$

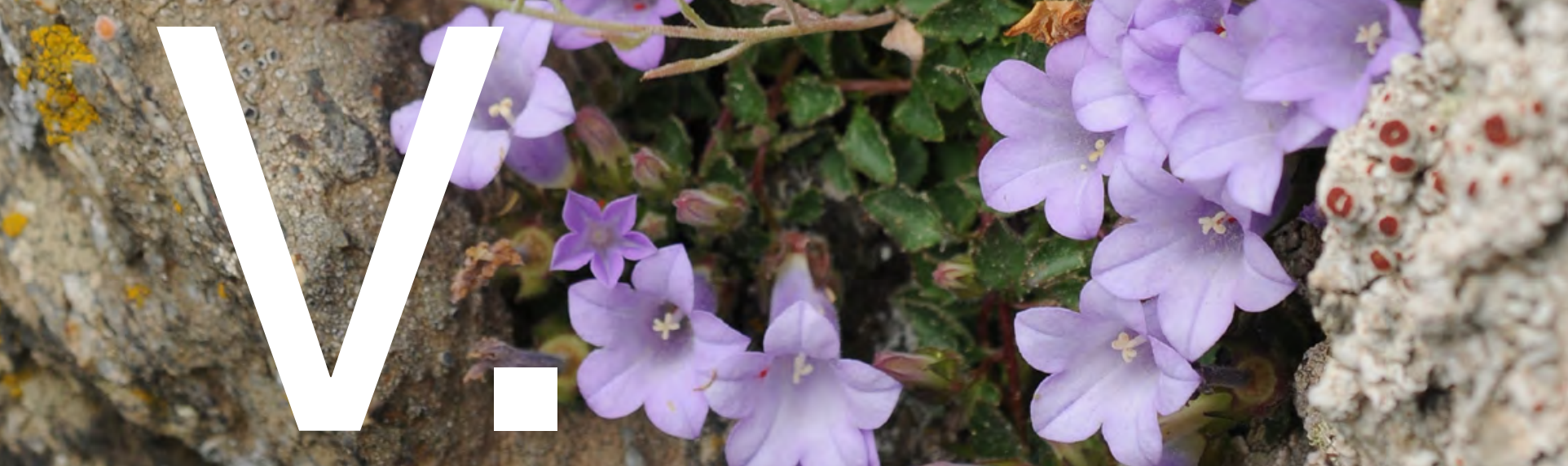

nes

ma $5=$

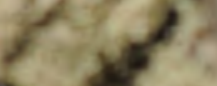

5 


\section{Procedures}

The following procedures are the main components of a Green Status assessment. These procedures are discussed in detail in the Background and Guidelines for the IUCN Green Status of Species, which will evolve over time to incorporate guidance on using new types of data that may become available, and new advances in analysis methods.

\section{Determining range}

\section{a. Determining indigenous range}

The indigenous range (see definition) is based on all known, inferred and (spatially) projected sites of occurrence, both current and historical. It includes conservation translocations that conform with IUCN Standards and Petitions Subcommittee (2019), but not vagrancies. For recovery objectives to be ambitious and aspirational, and to avoid shifting baselines, indigenous range should be based on the distribution at a date that is as early as feasible, while recognising that going too far back would increase uncertainties due to scarcity of data. Ideally, this date would be set to "a time before human beings were the most important element limiting species' distributions" (Sanderson, 2019). However, variation among species in terms of data availability and quality means that such a criterion would result in assessments that may not be comparable. Therefore, species in the same geographic region and under similar threats should have similar benchmark dates for determining indigenous range (Stephenson et al. 2019). Based on analysis of trends in historical data on habitat cover and human populations (from Klein Goldewijk et al., 2011), a default benchmark date of 1750 CE is appropriate in most regions and is in line with the IPCC definition of the start of the industrial era. This benchmark date can be modified (see Background and Guidelines), but should not be earlier than 1500 CE or later than $1950 \mathrm{CE}$.

\section{b. Determining expected additional range}

Expected additional range includes only those areas that are: (i) not part of the indigenous range; and (ii) are currently suitable or strongly likely to become suitable in the next 100 years; and (iii) are strongly likely to become occupied by the species in the next 100 years, with or without human assistance (provided those with human assistance conform with IUCN Standards and Petitions Committee (2019)). This likelihood can be assessed based on modelling (e.g. of climate-induced shifts), planned translocations, or empirical observation of range shifts.

Because of the time horizons commonly used to project range changes due to climate change, expected additional range would normally be used only for the long-term future (i.e. for calculating the Long-term Potential). Unless some SUs within the expected additional range can become occupied, or become focus of conservation efforts, in the next 10 years, short-term future scenarios (for calculating Conservation Dependence and Conservation Gain) do not have to consider expected additional range.

\section{c. Managed and introduced individuals}

For both indigenous and expected additional range, only 'wild' individuals of the species should be considered in determining the state in each spatial unit. For the definition of 'wild,' see the current version of the Red List Guidelines (IUCN Standards and Petitions Committee, 2019), section 2.1.4.

For both indigenous and expected additional range, wild subpopulations resulting from introductions outside the indigenous range of the species should be considered in determining the state, provided certain conditions are met (see the current version of the Red List Guidelines (IUCN, 2019), section 2.1.3). 


\section{Delineating spatial units}

Spatial units are used to incorporate representation, one of the three requirements of a Fully Recovered species (see II.3). A Fully Recovered species occurs as a set of functional populations across a representative set of ecosystems and communities across its range. A practical way of assessing this condition is to determine the state of the species in each of several spatial units that comprise its range, delineated to represent the variation of ecological conditions or settings within the range of the species. The spatial units must be chosen carefully because the Green Score is sensitive to the number of units. Because spatial units are valued equally in the calculation of Green Scores, they should be defined to represent areas of similar importance for the species' conservation, both with respect to overall representation and with respect to conservation action, across the full extent of the species' indigenous range and expected additional range.

Spatial units can be delineated by subpopulation, ecological and geographical features, and location, or a combination of these. Species-specific subdivisions based on species biology, such as subpopulations (defined in IUCN Standards and Petitions Committee (2019)), are preferred. Subspecies, stocks, genetic units, flyways, evolutionarily significant units, and discrete population segments are all conceptually related to IUCN's definition of subpopulation. Although not species-specific, divisions based on ecoregions, habitat types, or ecosystem types can also be used to define spatial units because they are defined based on ecological criteria and thus capture the different ecological settings in which a species exists or existed. Geographical features (e.g. watersheds, islands, lakes, mountain ranges) can be proxies for subpopulations. Recent fragmentation of the species into 'subpopulations' as a result of human activity is not an appropriate basis to delineate spatial units, if these 'subpopulations' were historically connected. Finally, areas defined by their vulnerability to a specific threatening process ('locations' in IUCN (2019)) can be used to define spatial units, on the assumption that the status of the species will be similar throughout an area that is similarly threatened.

Many restricted range species may be assessed based on a single spatial unit, or two spatial units (e.g. one for the extant range and one for the extirpated range). This may also be the case for a species that has always existed in a very specific type of ecosystem or a species whose function is similar in the different ecological settings it exists in. For other species, three or more spatial units may be necessary to represent the variety of ecological conditions and contexts that the species occurs or has occurred in.

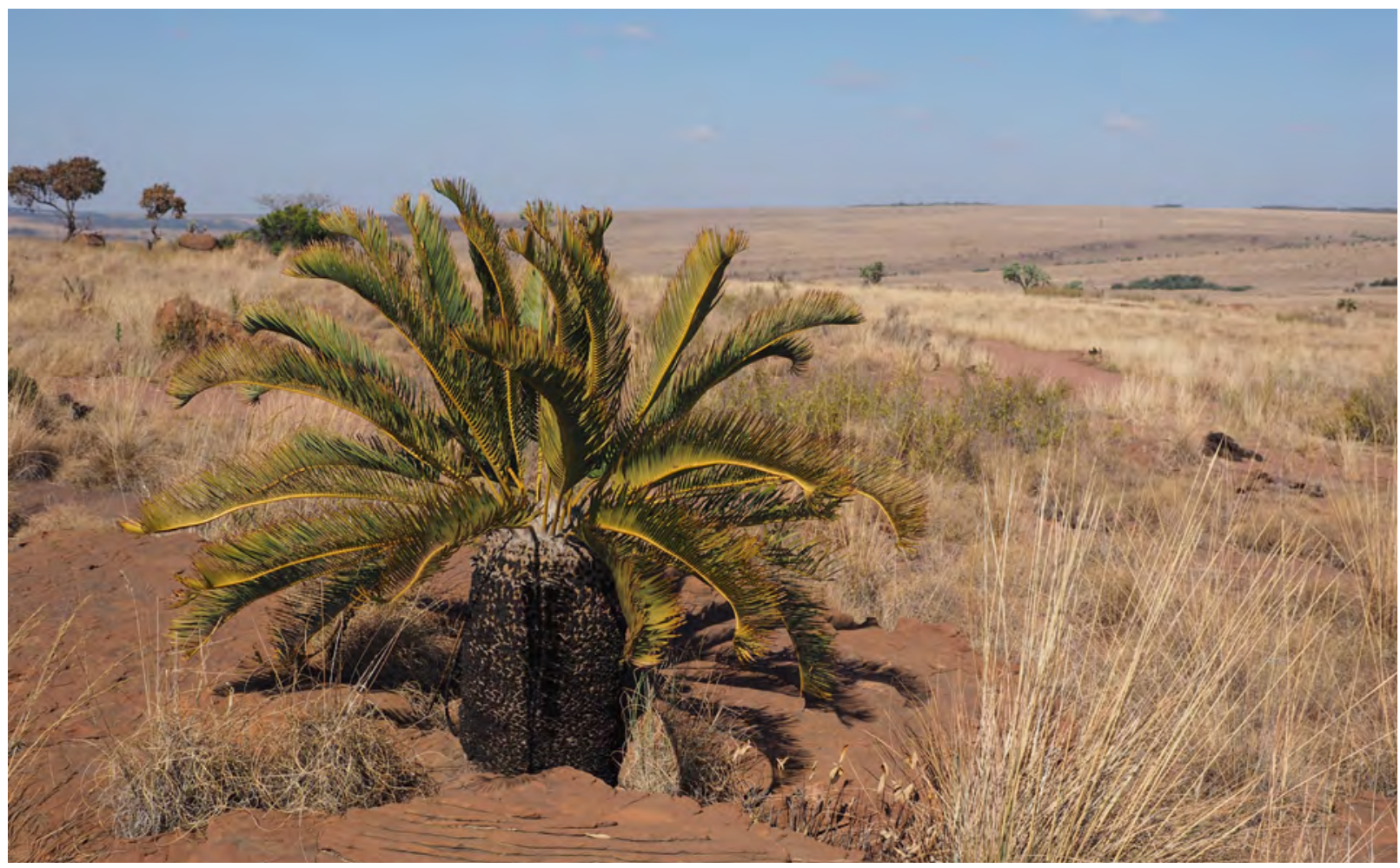

Woolly cycad (Encephalartos lanatus) @ Michael Calonje 


\section{Assessing the state in a spatial unit}

State in each spatial unit is assessed sequentially in the order Absent, Present, Viable, Functional. The definitions of Absent, Present, and Viable require the use of Red List categories; thus, the assessors should consult the latest versions of the Red List documents (IUCN, 2012a; IUCN, 2012b; IUCN Standards and Petitions Committee, 2019). Assessors should document the most likely state in each spatial unit as well as the minimum and maximum plausible states, to reflect the level of uncertainty. The guidance for Regional Red Listing should be used unless there is only one Spatial Unit for the whole species.

\section{a. Assessing Absence}

The species is Absent in a spatial unit if its Red List category in the spatial unit is Extinct (EX), Extinct in the Wild (EW), Regionally Extinct (RE), Critically Endangered (Possibly Extinct) (CR(PE)), or Critically Endangered (Possibly Extinct in the Wild) (CR(PEW)). For methods to determine the Extinct category vs. the Possibly Extinct tags, see the current version of the Red List Guidelines. The weight for Absent in Equation 1 is 0.

\section{b. Assessing Presence}

The species is Present in a spatial unit if it occurs (i.e. not Absent), and either (i) its regional Red List category in the spatial unit is threatened, that is, Vulnerable (VU), Endangered (EN) or Critically Endangered (CR), but not CR(PE) or CR(PEW), or (ii) the category is Near Threatened (NT) and the spatial unit population is undergoing 'continuing decline' in population size, as defined by IUCN Standards and Petitions Committee (2019). In spatial units that lack mature individuals, the presence of immature individuals (including seed banks) qualify the spatial unit as Present. Similarly, spatial units with extinction debt (i.e. lack of recruitment will cause eventual local extinction) are assessed as Present, not Absent. A regional Red List assessment (IUCN, 2012b) requires provisions for the possibility of rescue effect as a result of immigration from other spatial units, and may result in downlisting of the threat category, for example from VU to NT (see IUCN (2012b)). The state in the spatial unit should be based on the category after any such applicable adjustment to the threat category.

The default weight for Present in Equation 1 is 3. Assessors may choose instead to give the weight that corresponds to the specific regional Red List category for the population in the spatial unit: 1.5 for CR, 2.5 for EN, 3.5 for VU, or 4.5 for NT with continuing decline (see Background and Guidelines for an explanation of these fine-resolution weights). If the Red List category is DD, use the weights for Absent (zero) for the minimum value and Functional (9) for the maximum value.

\section{c. Assessing Viability}

The species is considered Viable in a spatial unit if a regional IUCN Red List assessment (IUCN, 2012b) of the species in that spatial unit results in a designation of Least Concern (LC); or a designation of Near Threatened (NT) and the spatial unit population is not undergoing 'continuing decline' in population size, as defined by IUCN Standards and Petitions Committee (2019). A regional Red List assessment (IUCN, 2012b) requires provisions for the possibility of rescue effect as a result of immigration from other spatial units, and may result in down-listing of the threat category, for example from NT to LC (see IUCN (2012b)). The state in the spatial unit should be based on the category after any such applicable adjustment to the threat category.

The default weight for Viable in Equation 1 is 6 . The assessors may choose instead to give the weight from the following list that corresponds to the specific regional Red List category in the spatial unit: 5.5 for NT without continuing decline, or 6.5 for LC. If the category is DD, use the weights for Absent for the minimum value and Functional for the maximum value. 


\section{d. Assessing Functionality}

Functionality, like viability, is assessed within each spatial unit. This requires assessing each spatial unit relative to functions that comprise the most important roles of the species. Although these functions may not be easy to determine, incorporation of functionality whenever possible is a critical element of an aspirational conservation vision. However, consideration of functionality in the context of species recovery should not be misinterpreted as prioritising conservation of species based on their functional importance.

Unlike the other states (Absent, Present, and Viable), Functional is not based on Red List criteria (but see section on the relationship of Viability and Functionality in Background and Guidelines). Functionality can be determined directly, by considering the interactions of the species and its contributions to ecosystem processes; or indirectly, by looking for symptoms of reduced functionality, analogous to the Red List approach of identifying symptoms of reduced viability (Akçakaya et al., 2020). When a function cannot be identified for a species, a number of proxies can be used to assess functionality, including population density or age structure in areas of low human impact or at a historical baseline.

Although, in principle, a population can contribute to a particular ecosystem process even when at a high risk of extirpation, for the purposes of the Green Status assessment process, the Functional score is only applied to spatial units that are also Viable. An exception is the rare case of a spatial unit with a naturally small ( $<1000$ mature individuals) population which is performing its ecological functions at baseline levels, but which would not meet the criteria for Viable because it would be assessed as VU under criterion D on the Red List. If such a spatial unit population is not declining, is not under a specific threat, and does not meet other criteria for VU, it may be assessed as Functional.

If the spatial units are based on subpopulations, then a spatial unit is scored as Functional if the subpopulation is Functional as defined above. If spatial units are not based on subpopulations and there are multiple subpopulations per spatial unit, a spatial unit is considered Functional if more than half of the subpopulations in that spatial unit are Functional (but if using finer-scale weights, the threshold is different; see below).

The default weight for Functional in Equation 1 is 9. The assessors may choose instead to give the weight from the following list that corresponds to the proportion of subpopulations within the spatial unit that were assessed as Functional: 8 for $<40 \%, 9$ for $40-70 \%, 10$ for $>70 \%$.

\section{Developing the Counterfactual Current scenario}

The Counterfactual Current scenario is an alternative present, envisaged in order to determine what the Current Green Score would have been had no conservation action taken place in the past. Developing the counterfactual scenario requires determining how the totality of all conservation actions from 1950 onwards has affected the population trajectory of the species across this time period, to be able to estimate what the state would have been today in each of the spatial units if those conservation actions had not taken place. Counterfactual thinking is a common tool in conservation (see Background and Guidelines for more details and instruction)

In developing the counterfactual scenario, the types of information to consider include population size and trends, changes in distribution and habitat availability, severity, scope and intensity of threats, and all conservation actions that have been put into effect since the start of main conservation actions. Conservation actions that were in place at 1950 and all that came after should be considered. Assessors should state the starting year of the past conservation actions considered in their assessment. In addition, assessors should consider information on the effectiveness of each type of conservation action within the broader context of changes in pressures and conservation opportunities for the species in the spatial unit.

If no past conservation action has been taken, then the counterfactual current scenario is the same as the current scenario. 


\section{Developing future scenarios}

A future scenario projects the future state in each spatial unit under different assumptions, for the purpose of calculating Conservation Gain, Conservation Dependence, and Recovery Potential metrics. The types of information to consider in developing future scenarios are similar to those for the Counterfactual Current scenario discussed above. The assessor should consider both current threats and plausible future threats. Future threats should be based on specific evidence (such as development plans, socioeconomic projections, etc.) and should not be speculative.

\section{a. Future-with-conservation}

For the Future-with-conservation scenario (for assessing Conservation Gain), the assessors should in addition consider the likely effects of all conservation interventions that are currently in place or are planned during the 10-year assessment window. However, assessors should not consider conservation actions that are thought of but not planned (e.g. if no clear aims, cost estimates, or time frames are specified), or conservation actions that are planned but are not expected to be initiated within the 10-year window. For planned actions, assessors need to make realistic assumptions about (i) the probability that the action will be implemented, and (ii) the probability that the conservation actions will have a positive effect on a species' population in a given spatial unit. For actions in place, the assessors should consider (ii). The likely benefits expected from these conservation measures should be discounted by these probabilities.

\section{b. Future-without-conservation}

For the Future-without-conservation scenario (for assessing Conservation Dependence), the assessors should consider the likely effects of all conservation interventions that are currently in place or are planned, and then remove their effects from the projections, similar to removing the effects of past conservation actions in a counterfactual scenario.

\section{c. Current Baseline}

The two future scenarios described in the previous sections (5a and 5b) are compared to the Current Baseline scenario in order to calculate the Conservation Gain and Conservation Dependence metrics. Current Baseline assesses the likely state of the spatial unit population after 10 years, based on current ongoing conservation action only (including actions which are highly likely to be implemented within one year, with funding and permissions in place), considering both current threats and plausible future threats. Future threats should be based on specific evidence (such as development plans, socioeconomic projections, etc.) and should not be speculative. The Current Baseline scenario accounts for the potential for ongoing decline or recovery of a spatial unit population regardless of planned conservation action or its withdrawal. If the assessors do not wish to calculate a Current Baseline, then by default it is taken to be the Current status.

\section{d. Long-term Potential}

For the Long-term Potential scenario (for assessing Recovery Potential), the assessors should envision the plausible conservation effort and innovation which could occur over the next 100 years. This includes actions which could be taken to eliminate threats and opportunities for habitat restoration and increased connectivity. This scenario must be realistic, considering the biological limitations of the species (e.g. generation time and maximum rate of population increase) and its habitat (e.g. rates of regeneration). It also needs to be realistic in terms of social, cultural and economic factors (e.g. projected trends in urbanization), but the long-term potential should not be limited by current political or budgetary constraints. This scenario is compared to the Current status (not the Current Baseline) in order to assess Recovery Potential. Long-term Potential is not meant to be an accurate prediction, because it would be impossible to accurately predict all the natural, social, economic, and technological changes to happen in the next 100 years. Rather, it is meant as a reasonable expectation of how much the species could recover, given what is known today. 


\section{Incorporating uncertainties}

Uncertainty about the state of the species (Absent, Present, Viable, Functional) in each spatial unit and for each scenario should be explicitly stated by specifying: (i) the lowest plausible state; (ii) the highest plausible state, and (iii) the most likely (best) state. These uncertainties are propagated to calculate the minimum and maximum values of the four conservation impact metrics (see Background and Guidelines, and Akçakaya et al. (2018) Appendix S1).

\section{Documenting assessments}

To ensure assessments are fully justified and to allow assessment data to be analysed, a set of minimum supporting information is required. These data facilitate transparency and repeatability and enable users to search and find information easily on the website. The Background and Guidelines for the IUCN Green Status of Species provides guidance on the following: (i) Required supporting information for all Green Status assessments; (ii) Required supporting information under specific conditions; and (iii) Recommended supporting information. Note that the Documentation Standards will be updated from time to time.

\section{Communicating assessment results}

How the results of an assessment are presented depends on who would use them and how. For some audiences, only one or two of the four conservation impact metrics may be relevant; for others all four can be presented. For most analyses involving multiple species, and for most research purposes, the numerical (percentage) values of the four conservation impact metrics, and their uncertainty bounds, should be used. For other purposes, the results may be communicated as a combination of these numerical results and categories, consistent with the categories and thresholds specified above (see 'Categories and thresholds,' IV.3).

\section{Regional (including national) assessments}

Green Status assessments at regional spatial scales are possible, but require careful considerations of the 'indigenous and expected additional range' and 'parts of the range' aspects of the definition of Fully Recovered. It is strongly recommended that regional assessments (including national assessments) are done only after the first two steps of the global assessments are completed: determining the indigenous and expected additional range, and delineating spatial units.

To the extent possible, the species' range considered in a regional assessment should involve one or more of the spatial units of the global assessment in their entirety. In other words, regional or national assessments should avoid dividing a spatial unit determined and delineated for the purposes of a global assessment. Including whole spatial units (of the global assessment) in regional assessments will make it possible to combine results of two or more regional assessments, and therefore facilitate the information flow from regional to global assessments. 


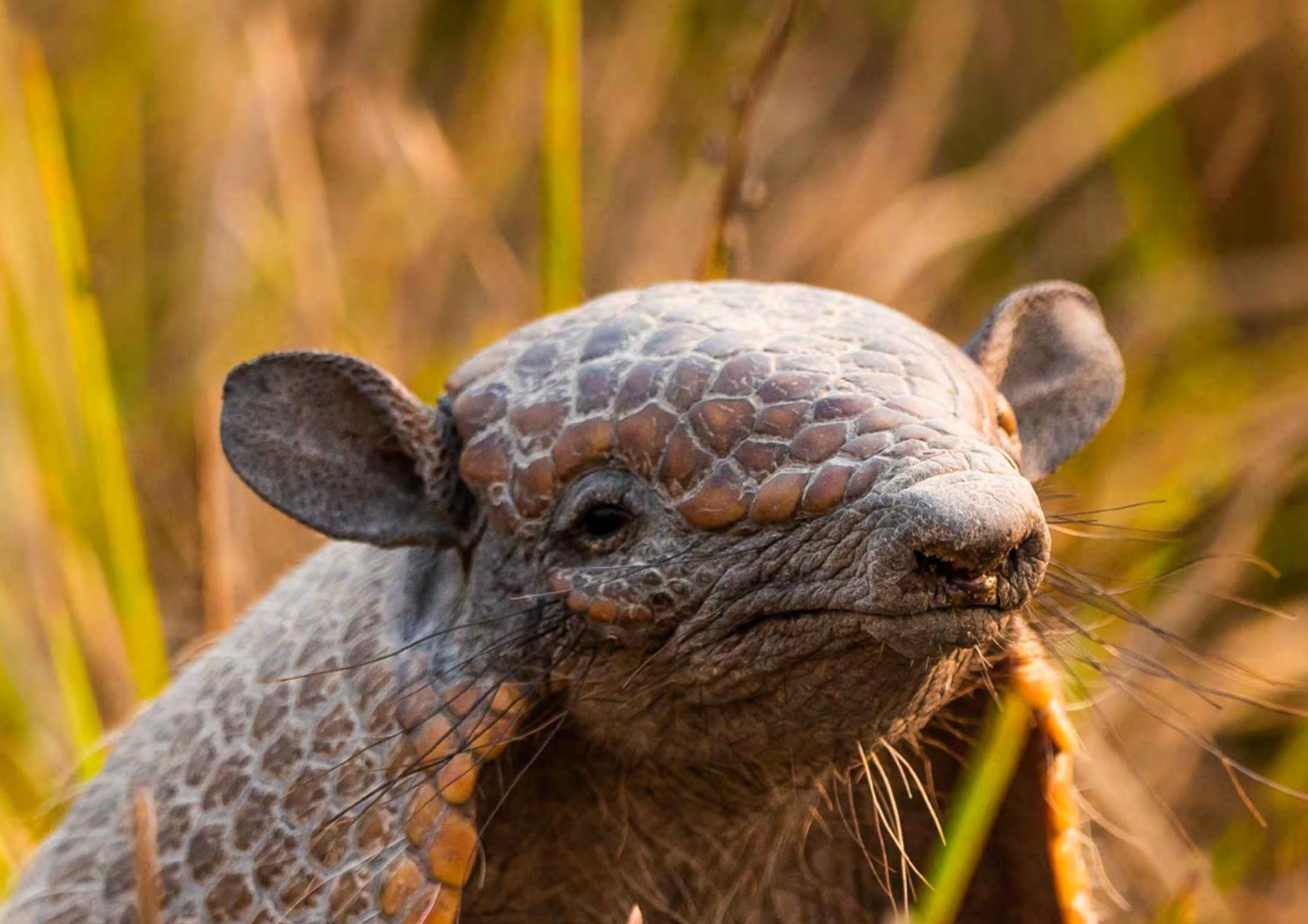

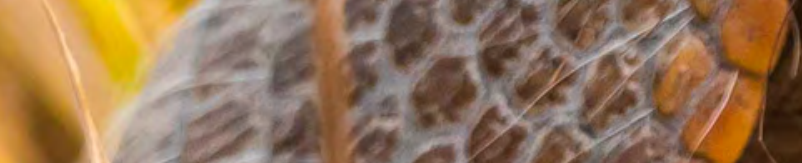

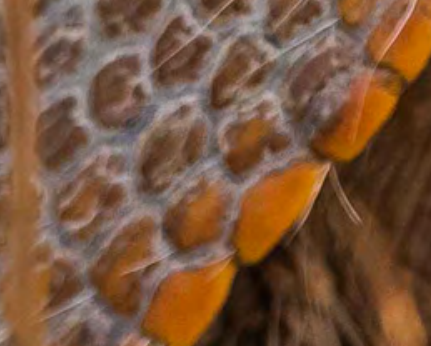

(1)

13

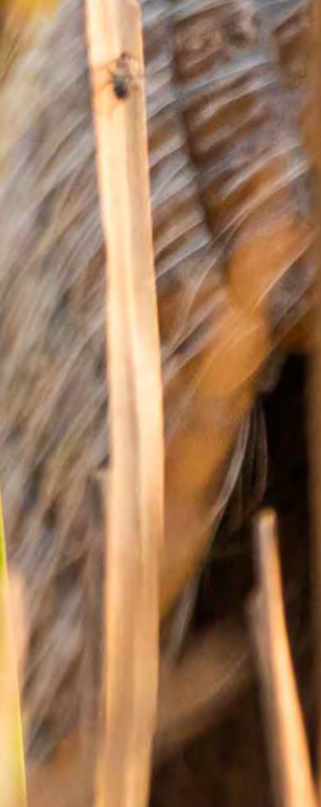




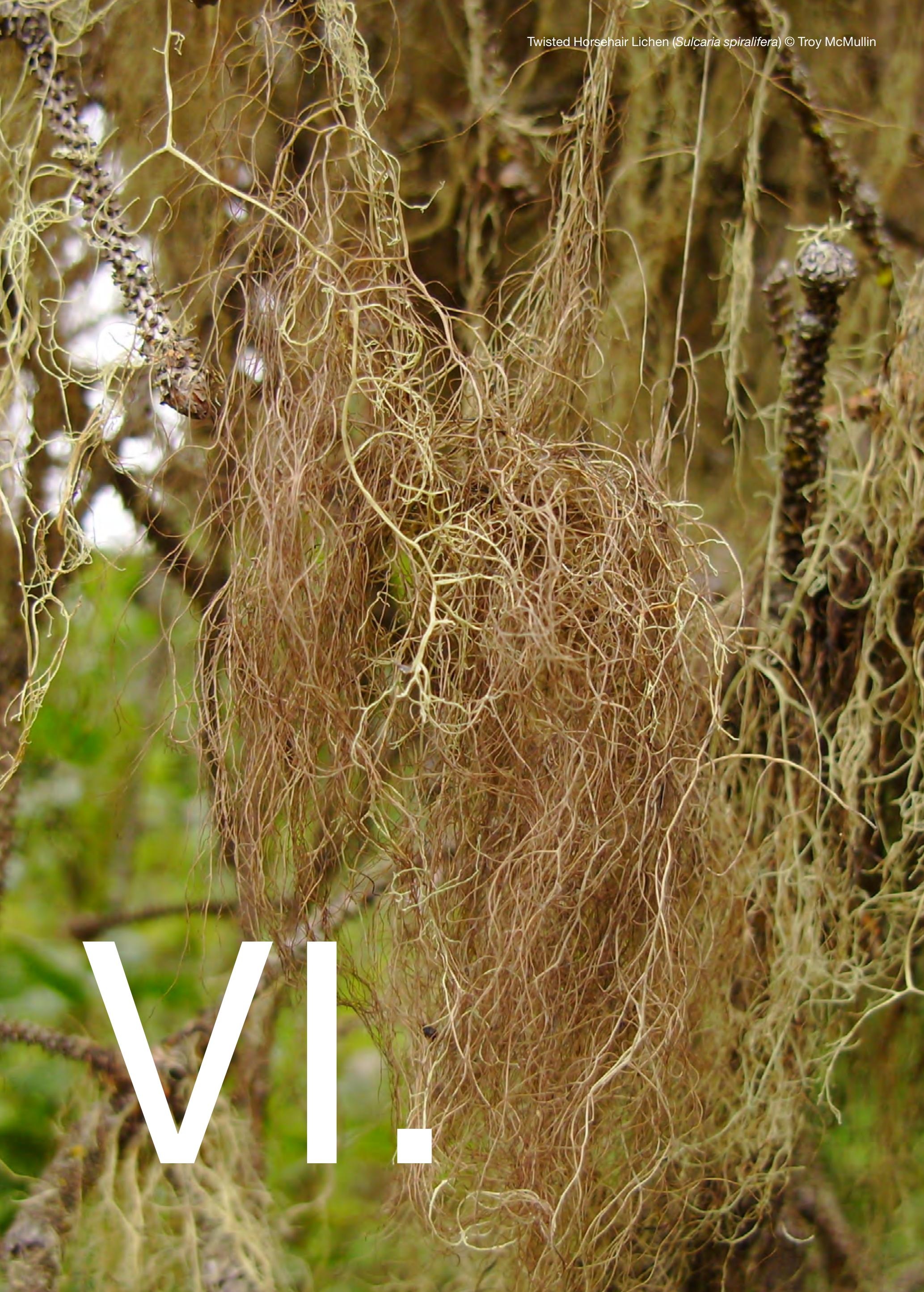




\section{References}

Akçakaya, H.R., Bennett, E.L., Brooks, T.M., Grace, M.K., Heath, A., Hedges, S., Hilton-Taylor, C., Hoffmann, M., Keith, D.A., Long, B., Mallon, D.P., Meijaard, E., Milner-Gulland, E.J., Rodrigues, A.S.L., Rodriguez, J.P., Stephenson, P.J., Stuart, S.N. and Young, R.P. (2018). 'Quantifying species recovery and conservation success to develop an IUCN Green List of Species'. Conservation Biology 32:1128-1138. Available at: https://doi.org/10.1111/cobi.13112 (Accessed: 10 September 2020).

Akçakaya, H.R., Rodrigues, A.S.L., Keith, D.A., Milner-Gulland, E.J., Sanderson, E.W., Hedges, S., Mallon, D.P., Grace, M.K., Long, B., Meijaard, E. and Stephenson, P.J. (2020). 'Assessing ecological function in the context of species recovery'. Conservation Biology 34:561-571. Available at: https://doi.org/10.1111/ cobi.13425 (Accessed: 10 September 2020).

Dudley, N. and Timmins, H.L. (eds.) (2021). A survey of user attitudes towards the proposed IUCN Green Status of Species. Gland, Switzerland: IUCN. https://doi.org/10.2305/IUCN.CH.2021.03.en

International Union for Conservation of Nature (IUCN). (2012a). IUCN Red List Categories and Criteria: Version 3.1. Second edition. Gland, Switzerland and Cambridge, UK: IUCN. Available at: https://portals.iucn.org/ library/node/10315

International Union for Conservation of Nature (IUCN) (2012b). Guidelines for Application of IUCN Red List Criteria at Regional and National Levels: Version 4.0. Gland, Switzerland and Cambridge, UK: IUCN. Available at: https://portals.iucn.org/library/node/10336

International Union for Conservation of Nature (IUCN) Species Survival Commission (SSC). (2013). Guidelines for Reintroductions and Other Conservation Translocations: Version 1.0. Gland, Switzerland: IUCN Species Survival Commission. Available at: https://portals.iucn.org/library/node/10386

International Union for Conservation of Nature (IUCN) Standards and Petitions Committee (2019). Guidelines for Using the IUCN Red List Categories and Criteria. Version 14. Prepared by the Standards and Petitions Committee. Downloadable from http://www.iucnredlist.org/documents/RedListGuidelines.pdf

International Union for Conservation of Nature (IUCN) and World Commission on Protected Areas (WCPA) (2017). IUCN Green List of Protected and Conserved Areas: Standard, Version 1.1. Gland, Switzerland: IUCN.

Klein Goldewijk, K., Beusen, A., van Drecht, G. and de Vos, M. (2011). 'The HYDE 3.1 spatially explicit database of human-induced global land-use change over the past 12,000 years'. Global Ecology and Biogeography 20:73-86. Available at: https://doi.org/10.1111/j.1466-8238.2010.00587.x (Accessed: 10 September 2020).

Sanderson, E.W. (2019). 'A full and authentic reckoning of species' ranges for conservation: response to Akçakaya et al. 2018'. Conservation Biology 33:1208-1210. Available at: https://doi.org/10.1111/ cobi.13399 (Accessed: 10 September 2020).

Stephenson, P.J., Grace, M.K., Akçakaya, H.R., Rodrigues, A.S.L., Long, B., Mallon, D.P., Meijaard, E., Rodriguez, J.P., Young, R.P., Brooks, T.M. and Hilton-Taylor, C. (2019). 'Defining the indigenous ranges of species to account for geographic and taxonomic variation in the history of human impacts: reply to Sanderson 2019'. Conservation Biology 33:1211-1213. Available at: https://doi.org/10.1111/cobi.13400 (Accessed: 10 September 2020). 




\section{IUCN}

INTERNATIONAL UNION

FOR CONSERVATION OF NATURE

WORLD HEADQUARTERS

Rue Mauverney 28

1196 Gland, Switzerland

mail@iucn.org

Tel +41 229990000

Fax +41 229990002

www.iucn.org

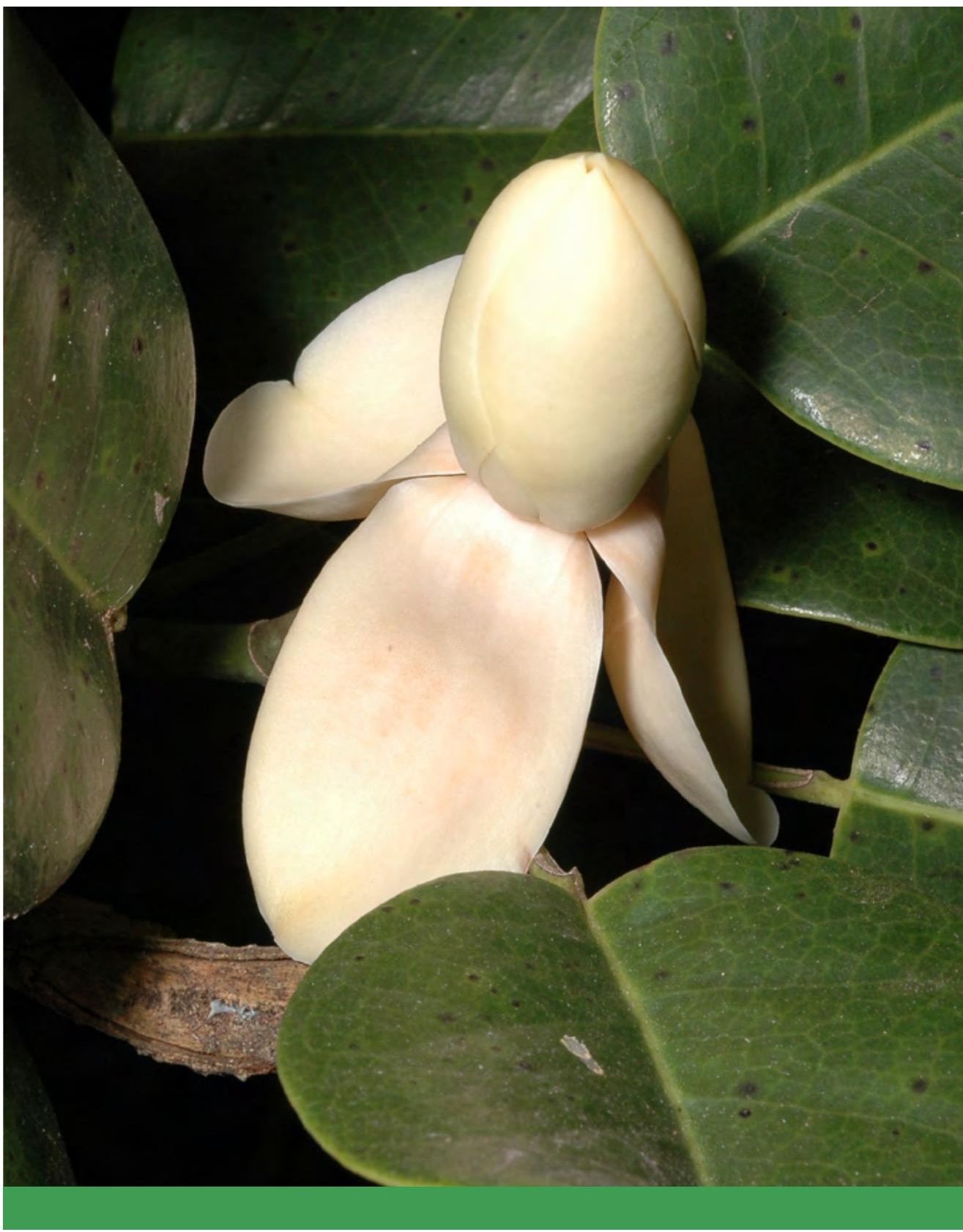

www.iucn.org/resources/publications 